REVIEW ARTICLE

\title{
Synthetic strategies of supported atomic clusters for heterogeneous catalysis
}

\author{
Hongpan Rong (10 ${ }^{1}$, Shufang $\mathrm{Ji}^{2 \otimes}$, Jiatao Zhang ${ }^{1 凶}$, Dingsheng Wang $\mathbb{1}^{2 凶} \&$ \\ Yadong $\mathrm{Li}^{2}$
}

Supported atomic clusters with uniform metal sites and definite low-nuclearity are intermediate states between single-atom catalysts (SACs) and nanoparticles in size. Benefiting from the presence of metal-metal bonds, supported atomic clusters can trigger synergistic effects among every metal atom, which contributes to achieving unique catalytic properties different from SACs and nanoparticles. However, the scalable and precise synthesis and atomic-level insights into the structure-properties relationship of supported atomic clusters is a great challenge. This perspective presents the latest progress of the synthesis of supported atomic clusters, highlights how the structure affects catalytic properties, and discusses the limitations as well as prospects.

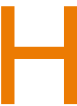

eterogeneous catalysts are widely used in industrial processes because they are easy to separate and recycle. Traditional heterogeneous nanocatalysts are usually prepared by impregnation or coprecipitation methods, resulting in a polydispersity in the number of atoms: nanoparticles, clusters, and even single atoms ${ }^{1}$. The size of metal species determines the fraction of surface atoms, which has a significant effect on both catalytic activity and selectivity. What is more, in the subnanometer size regime, the addition or removal of only one atom may bring about substantial influences on their catalytic properties ${ }^{2-6}$. Therefore, the traditional heterogeneous nanocatalysts are like a "black box", which makes the characterization and identification of active metal species a significant challenge. Furthermore, metal species with different sizes or structures may affect the adsorption and activation of substrates, triggering side reactions, thus decreasing the selectivity of catalysts.

The crucial first step to address these problems effectively is preparing heterogeneous catalysts with monodisperse metal species. Second, we have to identify the real active sites of catalysts systematically in the reaction process. Finally, a deep understanding of the relationship between their structures and properties presents a promising framework for the design and optimization of catalysts at the atomic scale.

Stemming from the unique geometric and electronic structures, supported atomic clusters with metal-metal bond and definite low-nuclearity metal active centers (especially the nuclearity of metallic atoms $<10$ ) can exhibit distinct catalytic properties ${ }^{1,7-9}$. In supported atomic clusters, most of the metal atoms are exposed as much as possible and are available for the reactant molecules. Therefore, supported atomic clusters have much more atom utilization efficiency in catalytic reactions than corresponding nanoparticles. In addition, due to the nanoparticles with the large size and complicated structure, the reactant molecules will be absorbed on uncertain

\footnotetext{
${ }^{1}$ Beijing Key Laboratory of Construction-Tailorable Advanced Functional Materials and Green Applications, School of Materials Science and Engineering, Beijing Institute of Technology, Beijing 100081, China. ${ }^{2}$ Department of Chemistry, Tsinghua University, Beijing 100084, China. ${ }^{凶}$ email: jisf15@mail.tsinghua.edu.cn; zhangjt@bit.edu.cn; wangdingsheng@mail.tsinghua.edu.cn
} 
sites of the surface of the nanoparticles, such as edge sites, corner sites, and planes, and formed different geometric structures with nanoparticles, resulting in a low catalytic selectivity. The investigation about supported atomic clusters with limited nuclearity provides a potential opportunity to reveal the real active sites in catalysis.

With mononuclear metal centers, single-atom catalysts (SACs) have drawn a broad interest in the field of catalysis. Many SACs have been synthesized by strategies including pyrolysis, external field-assisted method, ball milling, photochemical reduction, atomic layer deposition, etc ${ }^{10,11}$. With $100 \%$ atom utilization efficiency and strong metal-support interaction, SACs have shown outstanding performance in many chemical conversions, including thermochemical, electrochemical, and photochemical reactions $s^{12-15}$. However, in many critical industrial processes, especially in the catalytic reactions of multiple substrates, the single atomic active site cannot adsorb and activate multiple substrates at the same time, thus reducing the catalytic efficiency or even causing inactivity for the reaction (see Fig. 1$)^{16}$. In contrast, supported atomic clusters with two or more atoms can provide enough sites for the adsorption and activation of multiple substrates, which may endow a new catalytic pathway, decreasing the reaction barrier and improving the catalytic activity ${ }^{17}$. For example, for the selective oxidation reaction of alcohols to aldehydes, the calculated turnover frequency (TOF) of $\mathrm{Ru}_{3} / \mathrm{CN}$ is more than ten times higher than that of $\mathrm{Ru}_{1} / \mathrm{CN}^{18}$. Compared with SACs, supported atomic clusters not only provide more activation sites in each isolated catalytic site but also have different electronic structures due to the orbital overlapping between metal atoms. The synergistic effect among metal atoms for boosting catalytic performances is uniquely distinct in supported atomic clusters that contain two or more kinds of metal atoms. For instance, in terms of acid electrochemical oxygen reduction reaction (ORR), dual Fe-Co sites supported on $\mathrm{N}$-doped porous carbon exhibit higher activity than both Fe- and Co-SACs ${ }^{19}$. With the development of synthesis and characterization, supported atomic clusters will undoubtedly be the next key and hot topic.

With recent developments in both theoretical modeling and advanced atomic-resolution characterization technology, the concept of single-cluster catalysts has been popular in the past few years. Combing the atomic-resolution technologies and calculations, researchers can provide a clear image of the dispersion and geometry of the clusters, even their exact positions, and detailed local coordination environment. Unfortunately, the controlled synthesis of supported atomic clusters is considerably complicated because it is difficult to stabilize the precise numbers of atoms on the support rationally. As shown in Fig. 2, several synthetic strategies have been proven effective. In a long history, gas-phase synthesis and size-selected strategy was the only method to prepare supported metal clusters with precise numbers of atoms ${ }^{20-25}$. However, the throughput of this method is only enough for analytical-scale study and far from the requirement of the catalytic applications. Then, new strategies that can afford large-scale metal clusters were proposed. These synthetic methods include precursor-preselected strategy ${ }^{18,26,27}$, host-guest strategy ${ }^{19,28,29}$, wet chemical reduction ${ }^{30,31}$, dendrimerbased $^{32,33}$, and atomic layer deposition method ${ }^{34}$. Considerable scientists have focused on the support effects and dynamic behavior of supported atomic clusters during heterogeneous catalytic processes via in situ techniques and theoretical calculations ${ }^{35-38}$. Apart from synthesis and dynamism, extensive efforts have also been devoted to predicting the catalytic properties of supported atomic clusters by computational studies ${ }^{5,39-41}$. For example, firstprinciple theoretical studies show that $\mathrm{Fe}_{3} / \theta-\mathrm{Al}_{2} \mathrm{O}_{3}(010)$ and $\mathrm{Rh}_{1} \mathrm{Co}_{3} / \mathrm{CoO}(011)$ can catalyze the thermal conversion of $\mathrm{N}_{2}$ to $\mathrm{NH}_{3}$ via an associative mechanism ${ }^{42,43}$. The research of supported atomic clusters is in an eruption period.

In this perspective, we provide recent achievements in the synthesis of supported atomic clusters and discuss their structure-properties relationship from the atomic-level insights. Then we outline the prospects and challenges for the synthesis, characterization, and catalysis of supported atomic clusters.

\section{The size-selected strategy}

As noted above, the gas-phase synthesis and size-selected method have a long history, and numerous metal clusters with precise numbers of atoms have been prepared through this approach. Three steps, including the creation, separation, and landing of clusters, form the whole process. For the cluster generation, different techniques including laser vaporization (see Fig. 3a) ${ }^{44}$, magnetron sputtering ${ }^{45}$, cold or hot reflex discharge ion source ${ }^{46}$, arc discharge ${ }^{47}$, and electrospray ionization ${ }^{48}$ have always been used. However, clusters produced by these sources usually exhibit a wide distribution of sizes. To obtain charged metal clusters with well-defined composition and precise size, researchers have to do the size-selection process. Commonly utilized routes for sizeselection of clusters are electrostatic quadrupole mass filters ${ }^{49}$, radiofrequency quadrupole mass filters ${ }^{50}$, and time of flight ${ }^{51}$. To prevent the cluster fragmentation and substrate damage, researchers developed the landing technique from "hard landing" to "soft landing" 52,53 . Utilizing inert gas, or opposite electric field, the kinetic energy of the cluster can be decreased, and monodisperse samples can be produced ${ }^{21}$. Not only monometallic clusters, but this approach can also form binary alloy clusters ${ }^{54}$. Comprehensive reviews have been written comparing the advantages and disadvantages of each size-selection route in different catalytic applications ${ }^{37,55-57}$.
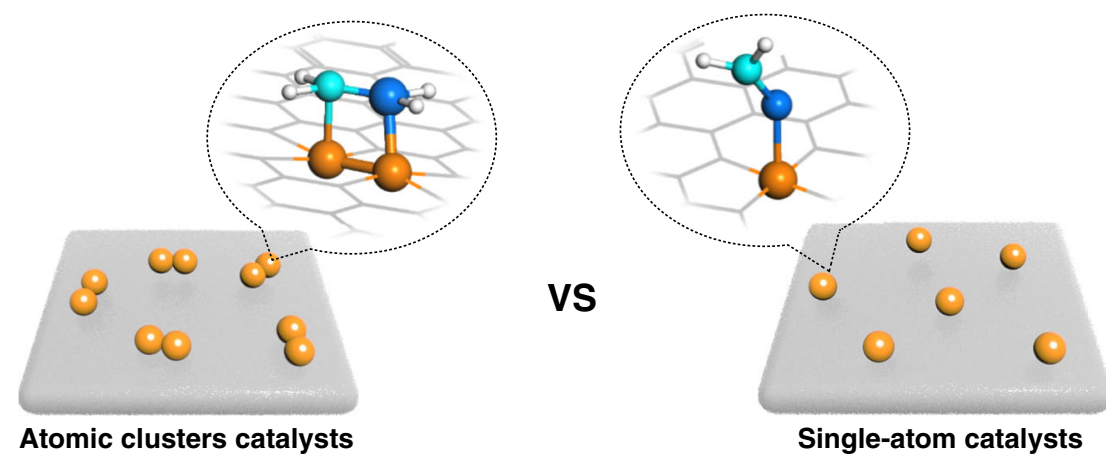

Fig. 1 Comparison diagram of adsorption modes in catalysis. Schematic view and corresponding structure model of the supported atomic clusters catalysts (left) and the single-atom catalysts (right). 


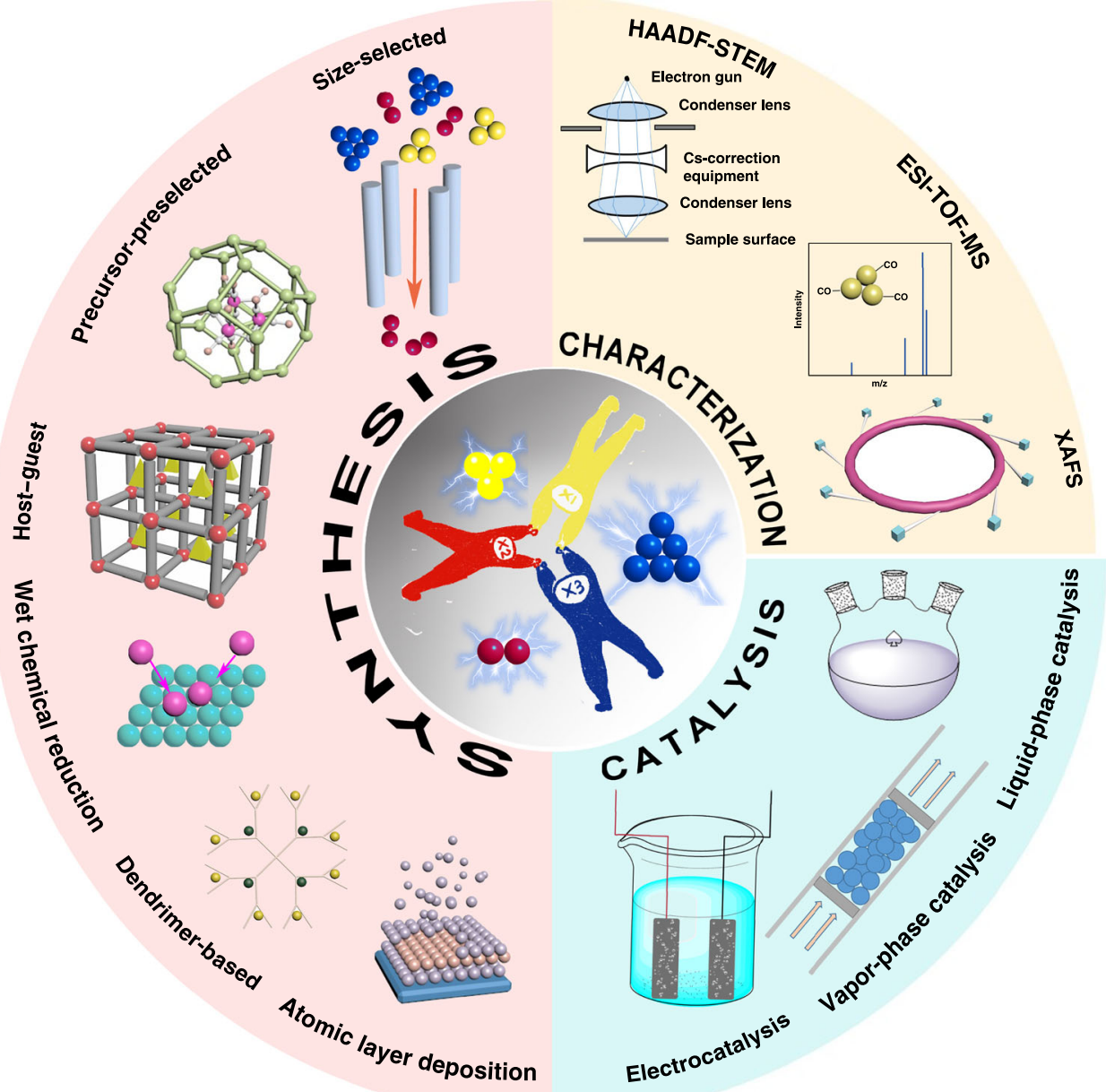

Fig. 2 Synthesis and catalytic performances of supported atomic clusters. Schematics for the size-selected, precursor-preselected, host-guest, wet chemical reduction, dendrimer-based, and atomic layer deposition strategies of supported atomic clusters; HAADF-STEM, ESI-TOF-MS, and XAFS are three important and typical characterization techniques; the electrochemical, vapor-, and liquid-phase catalytic performances of supported atomic clusters.

Due to its simplicity and significance, $\mathrm{CO}$ oxidation has been widely investigated ${ }^{1,7}$. Because of the high activity in the $\mathrm{CO}$ oxidation, in particular, for the low-temperature conditions, $\mathrm{Au}-$ based supported atomic clusters have attracted lots of attention. Compared with the Au SACs, Au supported atomic clusters showed higher activity in most cases ${ }^{58,59}$. In 2004, Anderson et al. studied the activity of soft-landed $\mathrm{Au}_{\mathrm{n}}$ clusters $(n=1,2,3,4$, and 7) on $\mathrm{TiO}_{2}$ for $\mathrm{CO}$ oxidation. They observed that $\mathrm{Au}_{3}$ showed substantial activity, while $\mathrm{Au}_{1}$ and $\mathrm{Au}_{2}$ did not catalyze $\mathrm{CO}$ oxidation $^{20}$. Size-dependent catalytic activity for $\mathrm{CO}$ oxidation has also been demonstrated over size-selected $\mathrm{Pt}_{n}$ and $\mathrm{Pd}_{n}$ supported atomic clusters ${ }^{60}$. The significant support effect on the catalytic performance should also be taken into account. Brune et al. studied the impact of the reduction state of the support on the $\mathrm{CO}$ oxidation over $\mathrm{Pt}_{7} / \mathrm{TiO}_{2}{ }^{61}$. The $\mathrm{Pt}_{7}$ clusters supported on highly reduced $\mathrm{TiO}_{2}$ showed the activity of two orders of magnitude lower than on the slightly reduced one. The decreased activity resulted from the oxygen consumption by the surface segregation of $\mathrm{Ti}^{3+}$ interstitials. However, comparisons were not made over supported atomic clusters with SACs in these reports. According to the report of Anderson et al. ${ }^{7}$, there is a good correlation between the catalytic activity and the Pd 3d X-ray photoelectron spectroscopy (XPS) shift, indicating the electronic structure of $\mathrm{Pd}_{\mathrm{n}}$ supported atomic clusters has a significant effect on the catalytic performances (see Fig. 3b). The Pd 3d binding energy varied non-monotonically with nuclearity number of supported atomic clusters. However, a stable valence electronic structure of Pd supported atomic clusters showed high $3 \mathrm{~d}$ binding energy, leading to low $\mathrm{CO}$ oxidation activity. By using $\mathrm{He}^{+}$ion scattering, Anderson et al. also found that the single-to-double layer geometric transition of the clusters was parallel with the change of catalytic activity. The geometric transition has also been observed in the cases of $\mathrm{Au}_{n}$ and $\mathrm{Pt}_{n}$ clusters in a similar nuclearity number range (about 10$)^{1,8}$. For the active sites of $\mathrm{Pd}_{n}$ supported atomic clusters for $\mathrm{CO}$ oxidation, the debate focuses on whether oxygen should be contained in the sites or not. Considerable experimental and theoretical results have supported the vital role of oxygen for the $\mathrm{CO}$ oxidation, especially when the host is the Ce-based materials ${ }^{62-65}$. When the support is amorphous $\mathrm{Al}_{2} \mathrm{O}_{3}, \mathrm{Pt}_{10}$, and $\mathrm{Ag}_{9} \mathrm{Pt}_{2,3}$ clusters exhibit turnover rate (TOR) of 3350 and $2500 \mathrm{CO}_{2}$ molecules/cluster/s at $300{ }^{\circ} \mathrm{C}$, respectively. The activation energies of $\mathrm{Ag}_{9} \mathrm{Pt}_{2,3}$ and $\mathrm{Pt}_{10}$ are around 50 and 60 $\mathrm{kJ} / \mathrm{mol}$, respectively ${ }^{66,67}$. By comparison, the apparent activation energies of Au clusters are between 25 and $30 \mathrm{~kJ} / \mathrm{mol}$. Although $\mathrm{Pt}_{n}$ and $\mathrm{Pd}_{\mathrm{n}}$ supported atomic clusters have exhibited high performance for $\mathrm{CO}$ oxidation, they can hardly surpass Au catalysts. Electronic (the energy level of the HOMO) and the geometric (the number of the layer) structure transition of all the clusters $\left(\mathrm{Au}_{\mathrm{n}}\right.$, $\mathrm{Pt}_{\mathrm{n}}$, and $\mathrm{Pd}_{\mathrm{n}}$ ) determined the catalytic activity of $\mathrm{CO}$ oxidation.

The dehydrogenation of alkanes, exceptionally light alkanes, to olefins is essential in the chemical industry. Since the reaction is endothermic, the process needs intense energy. Anderson et al. 

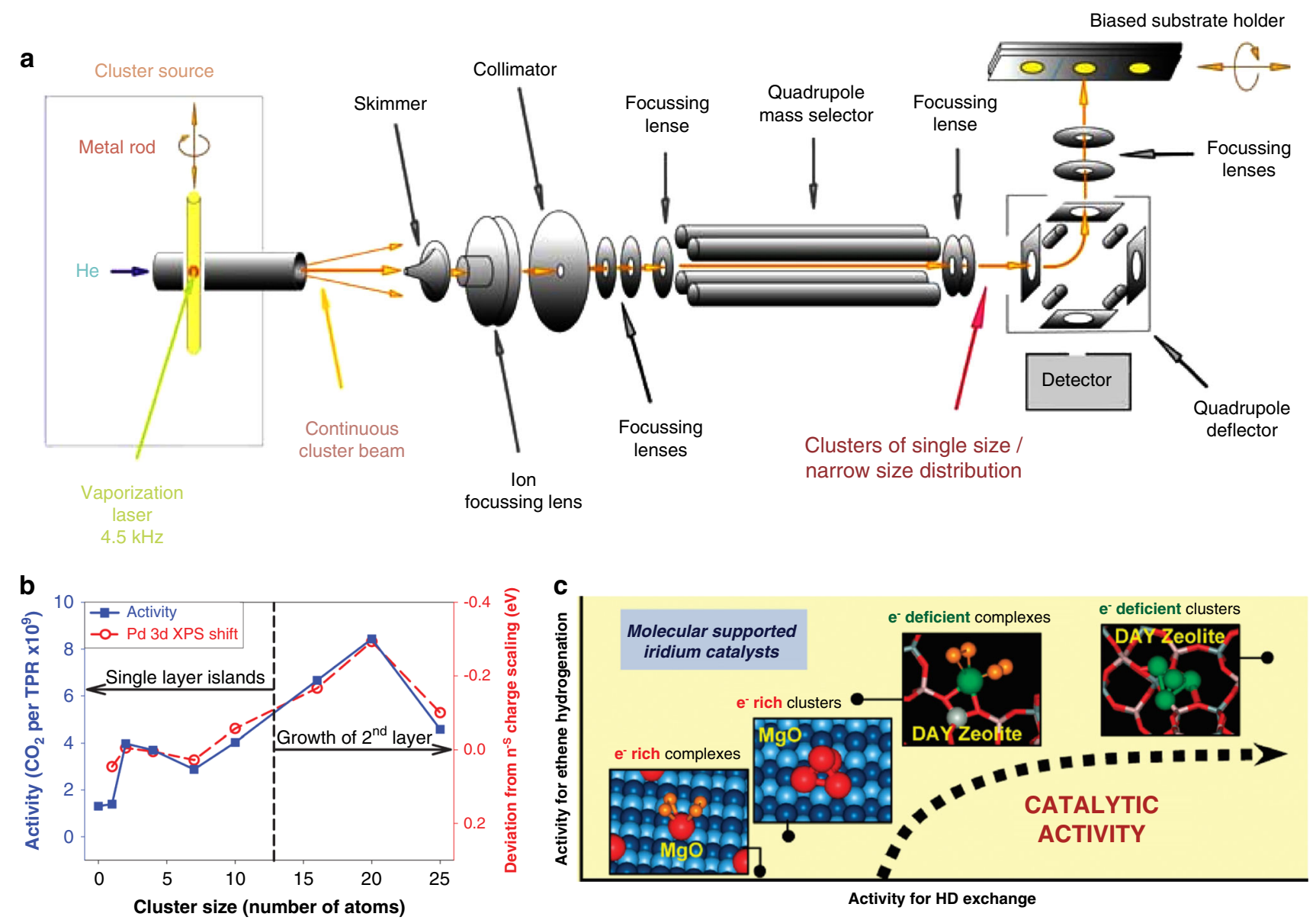

Fig. 3 The size-selected strategy. a Schematic of the cluster deposition setup, reprinted with permission from Springer Science+Business Media, Inc.: Springer Nature, Topics in Catalysis, ref. ${ }^{44}$ (Winans et al.). Copyright (2006). b CO oxidation activity observed during the temperature-programmed reaction (left axis, solid squares) compared with shifts in the Pd 3d binding energy, relative to expectations from smooth bulk scaling (right axis, open circles), as a function of cluster size. From ref. ${ }^{7}$. Reprinted with permission from the American Association for the Advancement of Science (AAAS). c Schematic illustration of mononuclear $\mathrm{Ir}$ and $\mathrm{Ir}_{4}$ clusters supported on $\mathrm{MgO}$ and dealuminated HY zeolite. Their relative catalytic activities for ethane hydrogenation are also presented, reprinted with permission from ref. ${ }^{71}$. Copyright (2011) American Chemical Society.

studied the size-selected $\mathrm{Pt}_{\mathrm{n}} / \mathrm{Al}_{2} \mathrm{O}_{3}(n=4,7$, and 8) for the dehydrogenation of ethylene to acetylene. With similar nuclearity numbers, $\mathrm{Pt}_{7}$ and $\mathrm{Pt}_{8}$ supported atomic clusters showed significant catalytic differences in this reaction, and $\mathrm{Pt}_{7}$ were more active than $\mathrm{Pt}_{8}$ and $\mathrm{Pt}_{4}$ supported atomic clusters. The geometric transition from single- to multi-layer, accompanied by changes of charge transfer and binding sites, accounts for the drop between $\mathrm{Pt}_{7}$ and $\mathrm{Pt}_{8}$ supported atomic clusters ${ }^{5}$. The reactions from olefins to epoxides are very crucial to the production of monomers in the polymer industry. Au- and Ag-based catalysts are always used for selective epoxidation of olefins ${ }^{68}$. The production of useless $\mathrm{CO}_{2}$ has long been the problem in the propene epoxidation catalyzed by conventional Ag catalysts. Vajda et al. reported that the sizeselected $\mathrm{Ag}_{3} / \mathrm{Al}_{2} \mathrm{O}_{3}$ supported atomic clusters could efficiently catalyze this reaction with a negligible amount of $\mathrm{CO}_{2}$ generation at a low temperature. Although higher temperature would cause the aggregation of $\mathrm{Ag}_{3}$ atomic clusters into $\mathrm{Ag}$ nanoparticles (NPs, ca. $3.5 \mathrm{~nm}$ ), the formed NPs exhibited enhanced selectivity towards propene oxide at temperatures lower than $120^{\circ} \mathrm{C}$ while maintaining a comparable activity to that of $\mathrm{Ag}_{3}$ supported atomic clusters ${ }^{23}$.

The hydrogenation of olefins is a well-known structureinsensitive reaction for platinum group metal nanocatalysts. However, when the size of the catalyst decreased to the subnanometric level, the structure-insensitive reaction changes to be structure-sensitive. Pérez-Ramírez et al. showed an inverse correlation between the catalytic activities and the degree of oxidation of Pd species for the semi-hydrogenation of 2-methyl-3butyn-2-ol ${ }^{69,70}$. Gates et al. studied the catalytic performance of $\mathrm{Ir}_{1}$ SACs and $\mathrm{Ir}_{4}$ supported atomic clusters on different kinds of supports $\left(\gamma-\mathrm{Al}_{2} \mathrm{O}_{3}, \mathrm{MgO}\right.$, and zeolite) for the hydrogenation of olefins ${ }^{71,72}$. Due to the new electronic structure/propensities of $\mathrm{Ir}_{4}$ clusters, which are not possessed by isolated atoms, the supported $\mathrm{Ir}_{4}$ atomic clusters showed higher catalytic activity than $\mathrm{Ir}_{1}$ SACs when the supports are the same. Further, they demonstrated that the extent of the nuclearity influence of the Ir species is closely related to the nature of supports. The activity gap between $\operatorname{Ir}_{1}$ and $\mathrm{Ir}_{4}$ species supported on $\mathrm{MgO}$ is much higher than that on zeolite. Besides, when the metal nuclearity was the same, the $\mathrm{Ir}_{4}$ atomic clusters supported on zeolite and $\gamma-\mathrm{Al}_{2} \mathrm{O}_{3}$ showed higher catalytic activity than $\mathrm{Ir}_{4}$ atomic clusters supported on the $\mathrm{MgO}$ (see Fig. 3c). Since $\mathrm{MgO}$ was more electron-donating than zeolite and $\gamma-\mathrm{Al}_{2} \mathrm{O}_{3}$, Ir sites were electron-rich when they were supported on it; the electron enrichment of Ir sites reduced the capacity of Ir to react with and form ligands from $\mathrm{C}_{2} \mathrm{H}_{4}$ and $\mathrm{H}_{2}$, hindering the activation of $\mathrm{H}_{2}$. Accordingly, the catalytic performances of metal atomic clusters can be significantly tuned by the nature of metal-support interactions.

Since the gas-phase synthesis and size-selected method, the only method to prepare supported atomic clusters in a long time, 
require ultrahigh-vacuum (UHV) conditions, the vast majority of catalytic researches of supported atomic clusters have focused on reactions under UHV conditions, which is far away from the realistic and industrial applications ${ }^{37}$. In the cases introduced above, scientists did these model catalytic reactions under UHV by adding a small volume of reaction gases. The catalytic mechanism and significant influence from cluster size and support effect on catalytic performance have been revealed. However, it is still a challenge to apply the synthesized atomic clusters catalysts to the actual catalytic reaction conditions to meet the industrial demand. Researchers have recently gained a series of achievements in studying the catalytic performances of supported atomic clusters under realistic conditions. Anderson et al. have studied atomic clusters supported on glassy carbon or indium tin oxide in UHV, and transferred them to an antechamber without air exposure. In this antechamber, the electrocatalytic performances of supported atomic clusters for oxygen reduction or ethanol oxidation can be explored ${ }^{73}$. Vajda et al. have focused on $\mathrm{UHV}$ and in situ reaction conditions of heterogeneous catalytic reactions, including dehydrogenation, epoxidation, and Fischer-Tropsch synthesis ${ }^{74,75}$. It is noteworthy that the gap between ideal UHV and realistic reaction conditions is not easy to be addressed. Some unexpected changes, such as the oxidation state of the cluster, reconstructions of support and clusters, and contaminants, may happen at increased pressures and temperatures. Therefore, it is a great challenge to construct a link between ideal UHV and realistic reaction conditions. Some efforts have been made to try to solve this problem. For example, Vajda et al. prepared two kinds of support for the size-selected $\mathrm{Pt}_{10}$ clusters: (1) a highly ordered alumina obtained under UHV, (2) amorphous alumina deposited on a silicon chip that is a similar model of real-world supports. They found both the $\mathrm{Pt}_{10}$ clusters and the support changed when $\mathrm{Pt}_{10} / \mathrm{UHV}$-alumina system was exposed to the realistic reaction conditions. The $\mathrm{Pt}_{10}$ clusters transformed from flat, two-dimensional configurations into three-dimensional configurations, and the ordered UHV-alumina was found to transform into an amorphous state ${ }^{67}$.

In a word, the size-selected method is effective to prepare monometallic atomic clusters with precise nuclearity; bimetallic and multimetallic clusters can also be prepared. However, the sophisticated equipment required for this method and the limited output are the major drawbacks.

\section{The precursor-preselected strategy}

The sintering of metal species can be described by the Ostwald ripening process, whose driving force is the different surface diffusion energy of metal atoms with different sizes. As a result, the sintering can be significantly suppressed if the size distribution of metal species is narrow. To prevent sintering during the synthesis of supported atomic clusters, the precursor-preselected strategy is advantageous as the metal species is homogeneous from the beginning to the end. The key to precursor-preselected strategy is the selection of metal precursors to obtain corresponding supported atomic clusters. For example, to prepare $M_{n}$ atomic clusters, we need to select or synthesize atom-precise, ligand stabilized metal molecule, or supramolecule $\left[\mathrm{M}_{n} \mathrm{~L}_{m}\right]$ as the precursor. Due to the diversity and the relatively easy removal of $\mathrm{CO}$, metallic cluster carbonyl had become the preferred precursor. In the 1970s and 1980s, scientists used metal cluster carbonyls as the precursor to prepare surface-supported metal clusters by decarbonylation at elevated temperatures ${ }^{26,76-82}$. Without atomic-resolution technologies, Infrared spectroscopy was a powerful characterization method to identify the structure of atomic clusters in these early studies. Although it was indicated that this approach could fabricate highly dispersed metal species, it was clear that the decarbonylation procedure is inevitably accompanied by cluster aggregation ${ }^{79}$.

Metal-organic frameworks (MOFs) with a porous structure can spatially confine metal complex and serve as tunable support for atomic clusters. As in the case of MOFs, to encapsulate and confine the metal cluster precursors, the size of the cages in MOFs should be suitable. The diameter of a metal cluster precursor is ideal between one of the pores and cages. As a sub-family of MOFs, zeolitic imidazolate frameworks (ZIFs) are prepared by the self-assembly method containing $\mathrm{M}-\mathrm{Im}-\mathrm{M}$ structures (where $\mathrm{M}$ stands for $\mathrm{Zn}, \mathrm{Co}, \mathrm{Cu}$ cations and $\mathrm{Im}$ stands for imidazolate linkers). As shown in Fig. $4 \mathrm{a}$, Wang et al. encapsulated $\mathrm{Ru}_{3}(\mathrm{CO})_{12}$ into the cages of ZIFs, followed by pyrolysis for forming $\mathrm{Ru}_{3}$ clusters stabilized on nitrogen-doped porous carbon $\left(\mathrm{Ru}_{3} / \mathrm{CN}\right)^{18}$. The $\mathrm{Ru}_{3}$ clusters were identified by aberration-corrected highangle annular dark-field scanning transmission electron microscopy (AC-HAADF-STEM, Fig. 4b). For the oxidation of 2aminobenzaldehyde, $\mathrm{Ru}_{3} / \mathrm{CN}$ showed one order of magnitude higher TOF than the $\mathrm{Ru}_{1} / \mathrm{CN}$ and $\mathrm{Ru}$ NPs (Fig. 4c). Theoretical calculations revealed that the distinct catalytic activity linked to adsorption energies and adsorption configurations of the 2aminobenzyl alcohol reactant on the three systems. When the $\mathrm{Ru}_{3}(\mathrm{CO})_{12} / \mathrm{ZIF}-8$ were reduced by hydrogen at a lower temperature, $\mathrm{Ru}_{3}$ clusters stabilized by ZIF-8 ( $\left.\mathrm{Ru}_{3} @ Z I F-8\right)$ were prepared due to the exceptional thermal stability of ZIF- $8^{83}$. In the semi-hydrogenation of terminal alkynes, Ru $\mathrm{u}_{3} @ Z I F-8$ exhibited higher activity and selectivity than $\mathrm{Ru}_{1} @ Z I F-8$ and $\mathrm{Ru}$ NPs@ZIF-8.

In addition, carbon nitride $\left(\mathrm{C}_{3} \mathrm{~N}_{4}\right)$ with abundant $\mathrm{N}$ sites can be employed to coordinate and stabilize the atomic metal clusters. For the synthesis $\mathrm{Fe}_{2}$ clusters $\left(\mathrm{Fe}_{2} / \mathrm{mpg}-\mathrm{C}_{3} \mathrm{~N}_{4}\right)$, bis(dicarbonyl cyclopentadienyl iron) $\left(\mathrm{Fe}_{2} \mathrm{O}_{4} \mathrm{C}_{14} \mathrm{H}_{10}\right)$ containing two $\mathrm{Fe}$ atoms in each molecule was selected as the precursor, and mesoporous $\mathrm{C}_{3} \mathrm{~N}_{4}$ support was employed to anchor the $\mathrm{Fe}_{2}$ species ${ }^{27}$. The $\mathrm{Fe}_{2} /$ mpg- $\mathrm{C}_{3} \mathrm{~N}_{4}$ exhibited outstanding catalytic performance for epoxidation of trans-stilbene (Fig. 4d). DFT calculations revealed that neither too strong (on Fe nanoparticles) nor too weak (on iron porphyrin) $\mathrm{Fe}-\mathrm{O}$ interactions could result in excellent catalytic activities.

In the case of $\mathrm{Pd}_{6} / \mathrm{CeO}_{2}$, Wang et al. selected $\mathrm{Pd}_{6} \mathrm{~L}_{4}$ supramolecule $(\mathrm{L}=1,3,5$-tris(4-pyridyl)-2,4,6-triazine) as the precursor of $\mathrm{Pd}_{6}$ clusters ${ }^{84}$. For the oxidation of benzyl alcohols, $\mathrm{Pd}_{1} /$ $\mathrm{CeO}_{2}$ showed extremely high activity, while $\mathrm{Pd}_{6} / \mathrm{CeO}_{2}$ was inert. DFT calculations revealed that owing to the larger size of $\mathrm{Pd}_{6}$ than $\mathrm{Pd}_{1}$, the $\mathrm{Pd}_{6}$ clusters impeded the interaction between hydroxyl groups of benzyl alcohols and $\mathrm{CeO}_{2}$ support, thus inhibiting the synergistic catalysis effect of $\mathrm{Pd}$ species and $\mathrm{CeO}_{2}$. These reports indicate the diversity of the nuclearity effect. The presence of neighboring metal atoms plays a vital role in the regulation of electronic structure. For some reactions, the regulation may lead to a positive change of the catalytic performance, but for other reactions, the change may be negative.

In addition, as shown in Fig. 4e, Pérez-Ramírez et al. fabricated $\mathrm{Pd}_{1}, \mathrm{Pd}_{2}$, and $\mathrm{Pd}_{3}$ clusters on $\mathrm{C}_{3} \mathrm{~N}_{4}$ by using $\mathrm{Pd}\left(\mathrm{NH}_{3}\right)_{4}\left(\mathrm{NO}_{3}\right)_{2}$, $\left[\mathrm{PdCl}\left(\mathrm{C}_{3} \mathrm{H}_{5}\right)\right]_{2}$, and $\left[\mathrm{Pd}(\mathrm{OAc})_{2}\right]_{3}$ as precursors, respectively ${ }^{4}$. They tested two kinds of reactions, including semi-hydrogenation of alkynes and Suzuki coupling, and found application-dependent nuclearity effects. For the selective hydrogenation of various functionalized alkynes, $\mathrm{Pd}_{3} / \mathrm{C}_{3} \mathrm{~N}_{4}$ was more active than $\mathrm{Pd}_{1} / \mathrm{C}_{3} \mathrm{~N}_{4}$ and $\mathrm{Pd}_{2} / \mathrm{C}_{3} \mathrm{~N}_{4}$ due to the reduced hydrogen activation barrier (Fig. 4f). In contrast, $\mathrm{Pd}_{1} / \mathrm{C}_{3} \mathrm{~N}_{4}$ surpass $\mathrm{Pd}$ supported atomic clusters in Suzuki coupling, exhibiting distinct chemoselectivity and high stability.

The examples listed above demonstrate the general applicability of precursor-preselected strategy to synthesize supported atomic clusters. As mentioned above, the most critical point for 
a

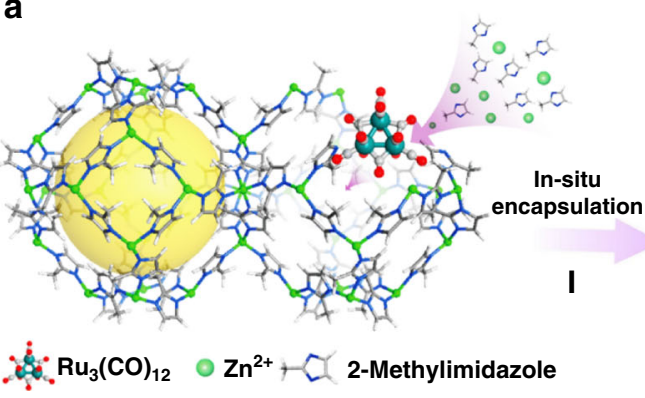

$\mathrm{Su}_{3}(\mathrm{CO})_{12} \odot \mathrm{Zn}^{2+}+\widetilde{2-M e t h y l i m i d a z o l e}$

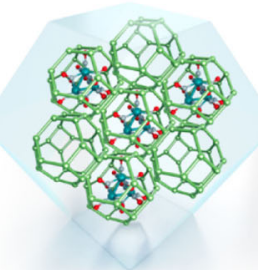

$\mathrm{Ru}_{3}(\mathrm{CO})_{12} @ \mathrm{ZIF}-8$

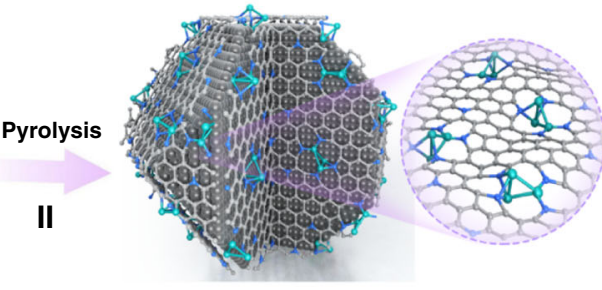

$\mathrm{Ru}_{3} / \mathrm{CN}$
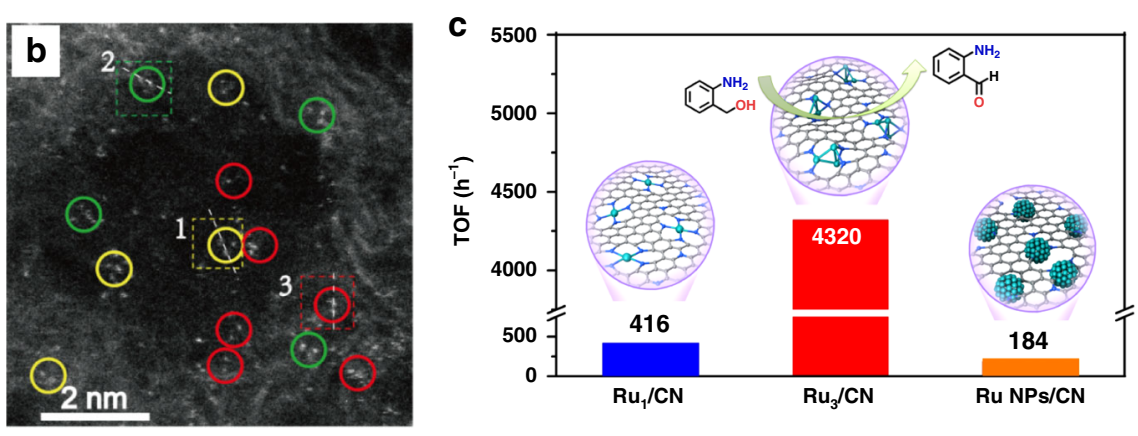

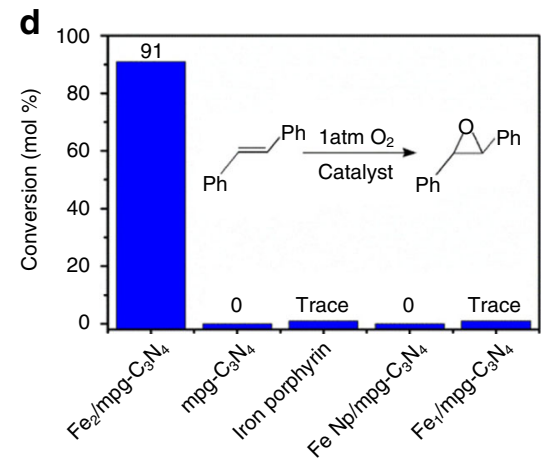

e

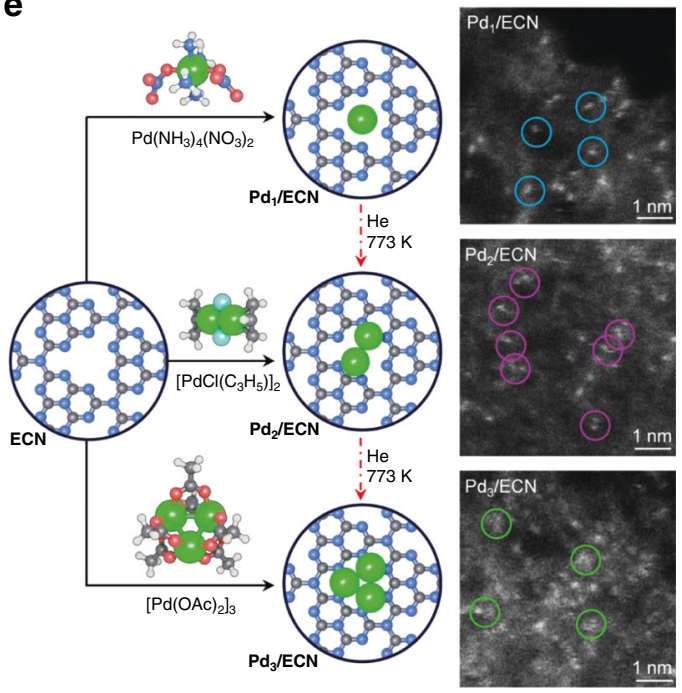

f

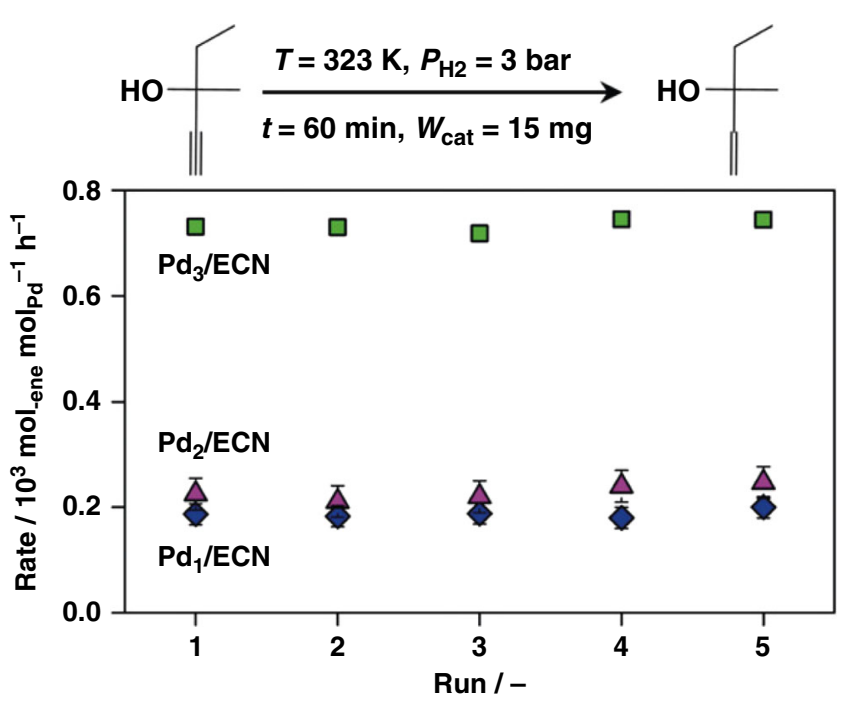

Fig. 4 The precursor-preselected strategy. a Illustration of the $\mathrm{Ru}_{3} / \mathrm{CN}$ preparation process. $\mathbf{b} \mathrm{AC}-\mathrm{HAADF}-\mathrm{STEM}$ images of $\mathrm{Ru} / \mathrm{CN}$. $\mathbf{c} \mathrm{TOFs}$ of the selective oxidation of 2-aminobenzaldehyde over the $\mathrm{Ru}_{3} / \mathrm{CN}, \mathrm{Ru}_{1} / \mathrm{CN}$, and Ru NPs/CN catalysts, reprinted with permission from ref. ${ }^{18}$. Copyright (2017) American Chemical Society. d Catalytic epoxidation of trans-stilbene over $\mathrm{Fe}_{2} / \mathrm{mpg}-\mathrm{C}_{3} \mathrm{~N}_{4}$ and other samples ${ }^{27}$. e Approaches to prepare low-nuclearity Pd catalysts based on carbon nitride and the corresponding AC-HAADF-STEM images of the resulting catalysts. Broken red arrows indicate unsuccessful routes. Selected Pd atoms (blue circles), dimers (pink circles), and trimers (green circles) are identified. $\mathbf{f}$ Rates of the hydrogenation reaction of 2-methyl3-butyn-2-ol during five runs. Adapted and reprinted with permission from ref. ${ }^{4}$. Copyright (2019) John Wiley \& Sons, Inc.

this strategy is the selection of metal precursors and proper support. So far, porous materials, such as MOFs and $\mathrm{C}_{3} \mathrm{~N}_{4}$, and oxides, such as $\mathrm{CeO}_{2}$, are candidate supports. We note that supported atomic clusters prepared by this strategy showed application-dependent nuclearity effects on the catalytic performances under realistic conditions. Since too weak interaction between the SACs and reactants usually leads to a high activation barrier, whereas too strong interaction between the nanoparticles and products blocks catalytic sites, both of which impair catalytic properties. On specific support, we can obtain intermediate interaction strengths by manipulating the nuclearity of supported atomic clusters and improve catalytic performances.

\section{The host-guest strategy}

The host-guest strategy based on reduction is advantageous in preparing supported atomic clusters, especially nitrogencoordinated dual-metal sites that contain heteroatoms. MOFs, a type of porous materials, are excellent platforms to confine and prepare atomic clusters in a controlled way. Pardo et al. synthesized the $\mathrm{Pd}_{4}$ clusters stabilized by $\mathrm{MOF}\left(\mathrm{Pd}_{4}-\mathrm{MOF}\right)$ through a three-step post-synthetic process (see Fig. $5 \mathrm{a}-\mathrm{d}$ ) ${ }^{85}$. In this process, initial MOF containing $\mathrm{Mg}^{2+}$ and $\mathrm{Cu}^{2+}$ cations were converted to a more robust MOF containing $\mathrm{Ni}^{2+}$ and $\mathrm{Cu}^{2+}$ cations by a transmetallation step; then partial $\mathrm{Ni}^{2+}$ cations were exchanged by $\left[\mathrm{Pd}\left(\mathrm{NH}_{3}\right)_{4}\right]^{2+}$, and $\mathrm{NaBH}_{4}$ was used as the 

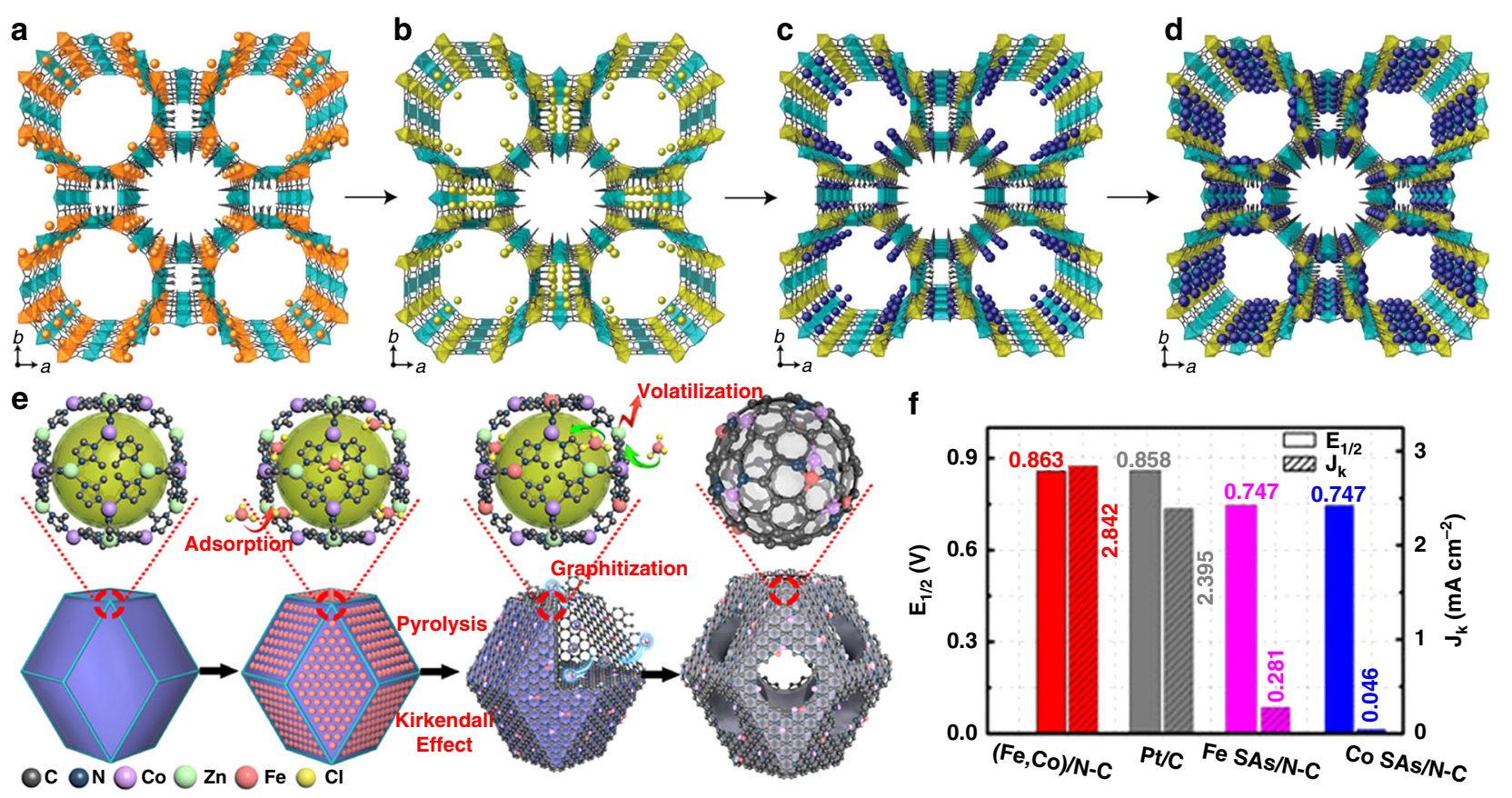

f
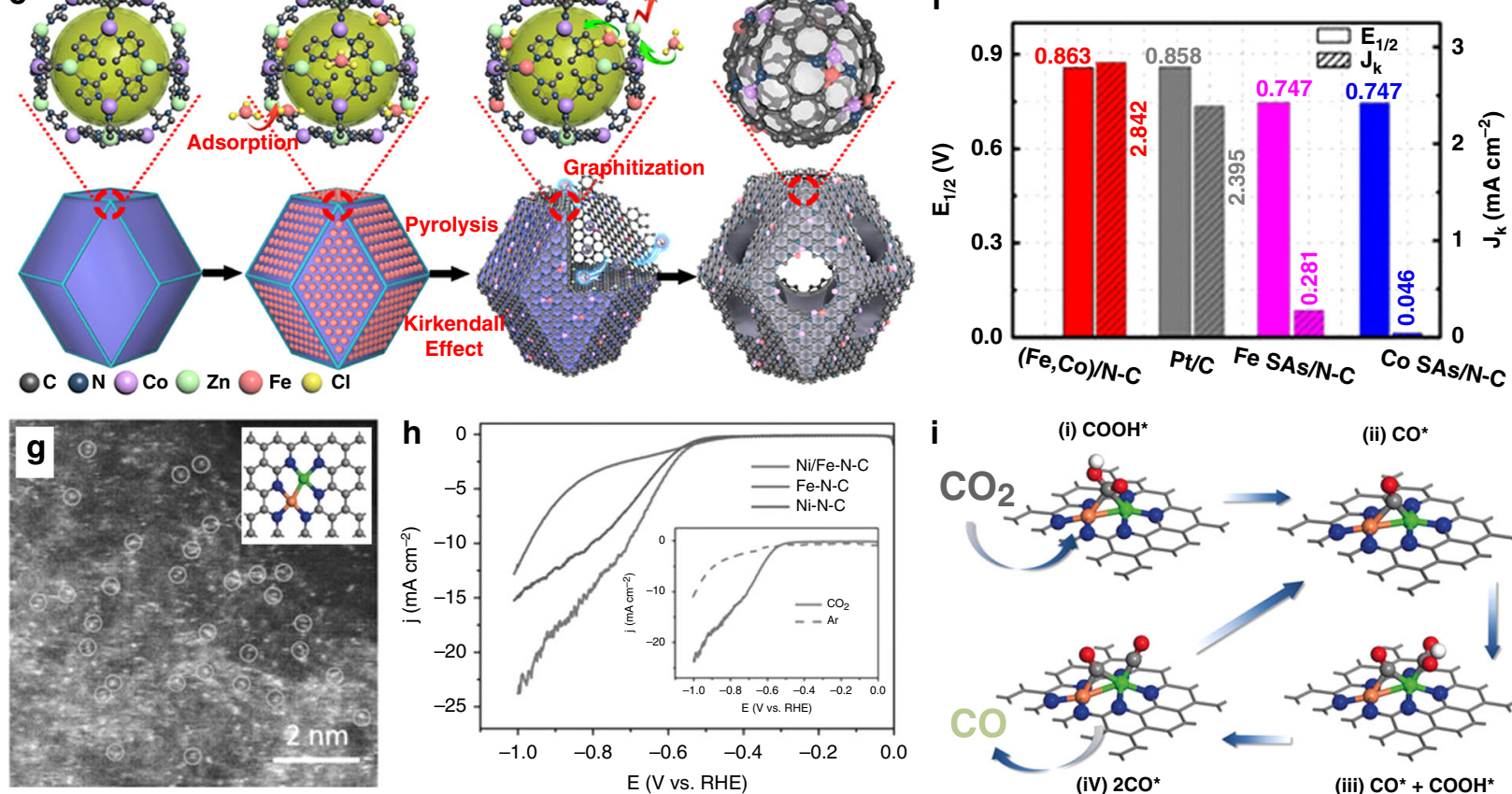

i

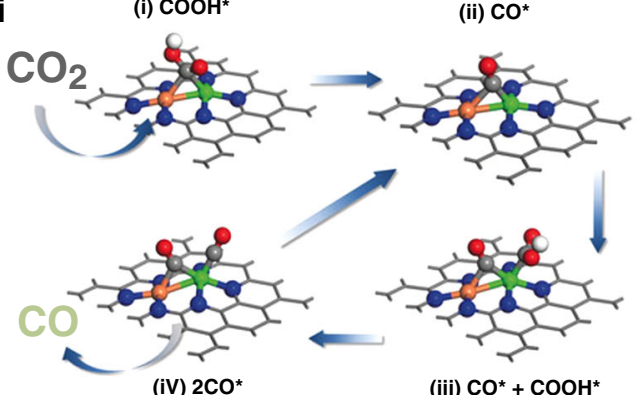

Fig. 5 The host-guest strategy. a-d Design approach showing the structures of 1-4 determined by single-crystal X-ray diffraction from the three-step post-synthetic process consisting of a transmetallation $\mathbf{1}$ (a) to give $\mathbf{2}(\mathbf{b})$, an exchange of the Nill cations of the pores by $\left[\mathrm{Pd}^{\prime \prime}\left(\mathrm{NH}_{3}\right)_{4}\right]^{2+}$ ones yielding $\mathbf{3}(\mathbf{c})$ and the final reduction process affording $\mathbf{4}$ (d). Reprinted by permission from Macmillan Publishers Limited: Springer Nature, Nature Materials, ref. 85 (Fortea-Pérez et al). Copyright (2017). e Preparation of ( $\mathrm{Fe}, \mathrm{Co}) / \mathrm{N}-\mathrm{C}$. $\mathbf{f}$ Comparison of $\mathrm{E}_{1 / 2}$ and $\mathrm{J}_{\mathrm{k}}$ for (Fe, Co)/N-C and other samples for ORR. Reprinted by permission from ref. ${ }^{19}$. Copyright (2017) American Chemical Society. $\mathbf{g}$ AC-HAADF-STEM images of Ni/Fe-N-C. The inset of (g) is the structure model of Ni-Fe dual atoms (Fe: orange; Ni: green; N: blue; C: gray). h Linear sweep voltammetry (LSV) curves for $\mathrm{CO}_{2}$ reduction over $\mathrm{Ni} / \mathrm{Fe}-\mathrm{N}-\mathrm{C}$ and other samples. Inset: LSV comparison for $\mathrm{Ni} / \mathrm{FeN}-\mathrm{C}$ in $\mathrm{Ar}$ - and $\mathrm{CO}_{2}$-saturated $0.5 \mathrm{M} \mathrm{KHCO}$ solution. $\mathbf{i}$ The catalytic mechanism on Ni/Fe-N site (based on the optimized structures of adsorbed intermediates $\mathrm{COOH}^{\star}$ and $\mathrm{CO}^{\star}$ ). Adapted and reprinted with permission from ref. ${ }^{28}$. Copyright (2019) John Wiley \& Sons, Inc. (Wiley).

reductant to give the final compound $\mathrm{Pd}_{4}-\mathrm{MOF}$. Substantial evidence for the structures of MOFs in different stages came from single-crystal X-ray diffraction measurement. The quasi-linear $\mathrm{Pd}_{4}$ clusters were stabilized by a synergic effect between the MOF network and the solvent molecules. The MOF-supported $\mathrm{Pd}_{4}$ atomic clusters outperformed state-of-the-art metal catalysts in carbene-mediated reactions of diazoacetates with high activity (yield $>90 \%$ ). Also, the $\mathrm{Pd}_{4}$ atomic clusters retained their catalytic activity in the flow reactions ( $>20$ cycles). Whereas a series of Pdbased salts, coordination compounds, and commercial Pd nanoparticles on different supports showed much lower catalytic activities (yield $<34 \%$ ) under the same conditions. In the next year, $\mathrm{Pt}_{2}$-MOF was synthesized via a similar process by the same Pardo group ${ }^{86}$. The specially designed MOFs are critical for the generation of the $\mathrm{Pd}_{4}$ and $\mathrm{Pt}_{2}$ clusters.

In 2017, Wu et al. used Co-doped ZIF-8 as the host and encapsulated $\mathrm{Fe}^{3+}$ within the cavities (see Fig. 5e) ${ }^{19}$. During the pyrolysis process, $\mathrm{Zn}^{2+}, \mathrm{Co}^{2+}$, and $\mathrm{Fe}^{3+}$ ions were reduced by asgenerated carbon, and $\mathrm{Zn}$ atoms were removed subsequently. The existence of dual-metal sites was proved by the AC-HAADFSTEM image. Combining XAFS and Mössbauer spectroscopic analysis, the presence of the $\mathrm{Fe}-\mathrm{Co}$ bond was confirmed. For the acidic ORR, the dual $\mathrm{Fe}$-Co sites supported on $\mathrm{N}$-doped porous carbon showed higher activity than both $\mathrm{Fe}$ - and Co-SACs (see Fig. 5f). At the same time, the dual-metal catalysts exhibited comparable onset potential and half-wave potential with commercial $\mathrm{Pt} / \mathrm{C}$. Also, the dual-metal catalysts were stable in an accelerated durability test with 50,000 cycles. DFT calculations reveal that the reduced activation barrier of the $\mathrm{O}-\mathrm{O}$ bond on dual-sites is crucial for the process of four-electron oxygen reduction. Recently, $\mathrm{Wu}$ et al. found the Fe-Co dual-metal catalysts could catalyze $\mathrm{CO}$ oxidation with $100 \%$ conversion at a low temperature $\left(-73^{\circ} \mathrm{C}\right)$, whereas the Co-SACs and Fe SACs showed CO conversions below $15 \%$ and 0 , respectively ${ }^{87}$. XANES spectra, pulse-adsorption microcalorimetry, and DFT studies showed that the $\mathrm{Fe}-\mathrm{Co}$ dual-metal sites catalyzed $\mathrm{CO}$ oxidation synergistically, with $\mathrm{CO}$ and $\mathrm{O}_{2}$ preferentially adsorbing at the Co and Fe sites, respectively. Very recently, Xing et al. also studied 
the enhanced ORR performance of $\mathrm{Fe}-\mathrm{Co}$ dual-atom sites. The $\mathrm{Fe}-\mathrm{Co}$ catalyst was also prepared by pyrolyzing $\mathrm{Fe}$-impregnated $\mathrm{ZnCo}-\mathrm{ZIF}^{88}$.

Inspired by these works, $\mathrm{Ni}-\mathrm{Fe}$ dual-metal sites were successfully prepared by using Fe-doped ZIF- 8 and $\mathrm{Ni}\left(\mathrm{NO}_{3}\right)_{2}$ as the host and guest, respectively ${ }^{28}$. XAFS and DFT simulations confirmed the existence of $\mathrm{Ni}-\mathrm{Fe}$ coordination. The Ni-Fe dual-metal catalyst prepared by pyrolysis also shows enhanced activity than the corresponding SACs $\left(\mathrm{Ni}-\mathrm{N}-\mathrm{C}\right.$ and $\mathrm{Fe}-\mathrm{N}-\mathrm{C}$ ) for $\mathrm{CO}_{2} \mathrm{RR}$ (see Fig. $5 \mathrm{~g}, \mathrm{~h}$ ). The $\mathrm{Ni}-\mathrm{Fe}-\mathrm{N}-\mathrm{C}$ exhibits a maximum $\mathrm{FE}_{\mathrm{CO}}$ of $98 \%$ at $-0.7 \mathrm{~V}$. Besides high activity, this dual-metal catalyst also displays outstanding stability for $\mathrm{CO}_{2} \mathrm{RR}$, maintaining high $\mathrm{FE}_{\mathrm{CO}}$ of $99 \%$ during $30 \mathrm{~h}$ of continuous electrolysis. DFT studies indicate that the bimetal-nitrogen sites undergo a geometric change into a COadsorbed moiety upon $\mathrm{CO}_{2}$ adsorption, which decreases the energy barrier for the formation of $\mathrm{COOH}^{*}$ and desorption of CO (see Fig. 5i).

Metallic bonding emerges when atomic orbitals on adjacent atoms overlap substantially. Supported atomic clusters containing several kinds of metal atoms (hetero-ACs) provide more opportunities for the manipulation of electronic structure than that containing only one kind of atom. At the same time, intercluster polarization and atomic interface effects in hetero-ACs may create remarkable catalytic properties. Upon different reagent molecules co-adsorbed on adjacent and different kinds of metal sites, the reaction barrier can be decreased, leading to exceptional high catalytic activity. The host-guest strategy is powerful to prepare hetero-ACs, and plenty of works are worth trying.

\section{The wet chemical reduction method}

Wet chemical reduction method was employed to prepare supported atomic clusters in which metal nanomaterials behaved as the supports for the clusters. Chen et al. used the Te nanowires as partial sacrificial templates to obtain $\mathrm{Cu}$-loaded $\left(\mathrm{Cu}_{4}\right.$ clusters) PdTe nanowires (see Fig. 6a) ${ }^{30} . \mathrm{NaOH}$ and $\mathrm{HCl}$ solutions were used to wash the solid product in sequence. On this nanowires, stable $\mathrm{Cu}_{1}{ }^{0}-\mathrm{Cu}_{1} \mathrm{x}+$ pair in $\mathrm{Cu}_{4}$ clusters was the essential catalytic active site for the electrochemical reduction of $\mathrm{CO}_{2}$, which was proved by DFT calculation and experimental and fitting XAFS spectroscopy. The stable $\mathrm{Cu}_{1}{ }^{0}-\mathrm{Cu}_{1} \mathrm{x}+$ pair in $\mathrm{Cu}_{4}$ clusters can selectively catalyze the $\mathrm{CO}_{2} \mathrm{RR}$ (see Fig. 6b). The Faradaic efficiency for $\mathrm{CO}\left(\mathrm{FE}_{\mathrm{CO}}\right)$ is above $92 \%$ under a relatively low potential $(-0.78 \mathrm{~V})$. Theoretical calculations reveal that the $\mathrm{Cu}_{1}{ }^{0}-\mathrm{Cu}_{1} \mathrm{x}+$ pair directly participate in the $\mathrm{CO}_{2} \mathrm{RR}$. At the same time, the other two $\mathrm{Cu}$ atoms in the $\mathrm{Cu}_{4}$ clusters stabilize the $\mathrm{Cu}$ clusters (see Fig. 6c). According to the theoretical calculations, Chen et al. have predicted stable $\mathrm{Cu}$ dimer supported by $\mathrm{C}_{2} \mathrm{~N}$ can exhibit high selectivity for $\mathrm{CH}_{4}$ with a small limiting potential $(-0.23 \mathrm{~V})$. They also forecast $\mathrm{C}_{2} \mathrm{H}_{4}$ can also be produced on the $\mathrm{Cu}_{2} @ \mathrm{C}_{2} \mathrm{~N}^{89}$. Different pathways take place for the formation of $\mathrm{CO}$, formic acid, hydrocarbons $\left(\mathrm{C}_{2+}\right)$, and multicarbon oxygenates. The $\mathrm{C}-\mathrm{C}$ coupling step is vital for the production of the latter two kinds of species.

Chen et al. prepared $\mathrm{Pt}_{3}$ decorated Co@Pd nanocatalyst by reducing $\mathrm{Co}, \mathrm{Pd}$, and $\mathrm{Pt}$ in sequence ${ }^{31}$. In this method, the short reduction time of $\mathrm{Pt}(10 \mathrm{~s})$ was vital for the formation of $\mathrm{Pt}_{3}$ clusters. Since the theoretical average coordination number of $\mathrm{Pt}-\mathrm{Pt}$ bond was 2.0, and the fitting XAFS data of this Pt decorated nanocatalyst was 1.95 , indicating the presence of $\mathrm{Pt}_{3}$ clusters. Compared with carbon supports, the metal nanomaterials show much brighter contrast in HAADF-STEM images, and it is difficult to identify the metal clusters via this visible characterization technique. For ORR in alkaline aqueous solution, the unique charge localization induced by $\mathrm{Pt}_{3}$ decoration on Co@Pd nanocatalyst resulted in a distinct mass activity, which is more than 30 times higher than that of commercial $\mathrm{Pt} / \mathrm{C}$.

The wet chemical reduction method is practical for the production of industrial-scale quantities of catalysts. However, the clusters prepared by this method usually have a broad size range. Capping ligands on nanostructures play a vital role in controlling morphology and prevent agglomeration. In some instances, the ligands are likely to impact the oxidation states of the clusters and the access of the reagent molecules. Therefore, there are limitations to the precise synthesis of supported atomic clusters by wet chemical reduction method.

\section{The dendrimer-based strategy}

$\mathrm{N}$-doped carbon materials are widely used to support and stabilize the atomic clusters via ligand protection from $\mathrm{N}$ coordinations. Dendrimers, macromolecules with a tree-like structure, contain a high proportion of N. At the same time, they have welldefined structures, as analogous to MOFs. Before MOFs, the dendrimers behaved as the nanosized reactor in which to synthesize various kinds of supported atomic clusters ${ }^{51,90-92}$. Dickson et al. reported the synthesis of $\mathrm{Ag}_{\mathrm{n}}$ clusters using a PAMAM G2 dendrimer (second generation of poly-(amidoamine) dendrimer) by a photochemical reduction method ${ }^{32}$. However, the clusters prepared were determined to be a mixture of $\mathrm{Ag}_{2}, \mathrm{Ag}_{3}$, and $\mathrm{Ag}_{4}$ by electrospray ionization mass spectrum (ESI-MS). When they reduced $\mathrm{Au}$ ions in PAMAM by using a chemical reducing agent $\left(\mathrm{NaBH}_{4}\right)$, Au nanoparticles and $\mathrm{Au}_{8}$ clusters were prepared. They removed larger Au nanoparticles through centrifugation ${ }^{90}$. It is interesting to note that only the $\mathrm{Au}_{8}$ cluster encapsulated by PAMAM was observed by ESI-MS. This work is a breakthrough for the synthesis of a metallic cluster with an atomic precise in the solution phase.

Besides PAMAM, scientists also prepared metal clusters using DPA (dendritic phenyl azomethine) ${ }^{92,93}$ and PPI (polypropylene imine $)^{94}$ dendrimers as host. Taking advantage of the basicity gradient of DPA dendrimer, scientists can prepare multimetallic nanoclusters precisely ${ }^{95,96}$. The supported atomic clusters prepared by the chemical dendrimer-based method also show sizedependent catalytic performance. Yamamoto et al. stabilized the $\mathrm{Pt}_{12}$ and $\mathrm{Pt}_{13}$ clusters by the DPA-TPM (fourth-generation DPA with a tetraphenylmethane core) and DPA-PyTPM (fourth-generation DPA with a triphenylpyridylmethane core), respectively. For acidic ORR, the $\mathrm{Pt}_{12}$ atomic clusters showed more than twofold mass activity compared with that of the $\mathrm{Pt}_{13}$ atomic clusters (see Fig. 6d). DFT analyses suggested that the different atomic coordination of $\mathrm{Pt}_{12}$ (deformed) and $\mathrm{Pt}_{13}$ (icosahedral) caused different catalytic activity ${ }^{2}$. Thus, by influencing the atomic coordination, the metal nuclearity can determine the catalytic performance significantly.

It should be noted that different dendrimers showed different capacities of encapsulation and stabilization, which influenced the catalytic performance consequently ${ }^{97}$. Due to the limited restriction capacity of dendrimers, the nuclearity of supported atomic clusters prepared by this method always larger than $10^{33}$ As a result, the studies for the catalytic performance of supported atomic clusters with nuclearity smaller than 10 are few. On the other hand, taking advantage of phenyl azomethine dendrimer's basicity gradient, scientists can precisely prepare multimetallic nanoclusters. ${ }^{95}$ However, the dendrimers used in these reports are mainly synthesized in the lab, limiting the scalable production of the atomic cluster by this method.

\section{The atomic layer deposition method}

The atomic layer deposition (ALD) method is robust in preparing $\mathrm{M}_{2}$ atomic clusters supported on the defect-rich carbon 
a
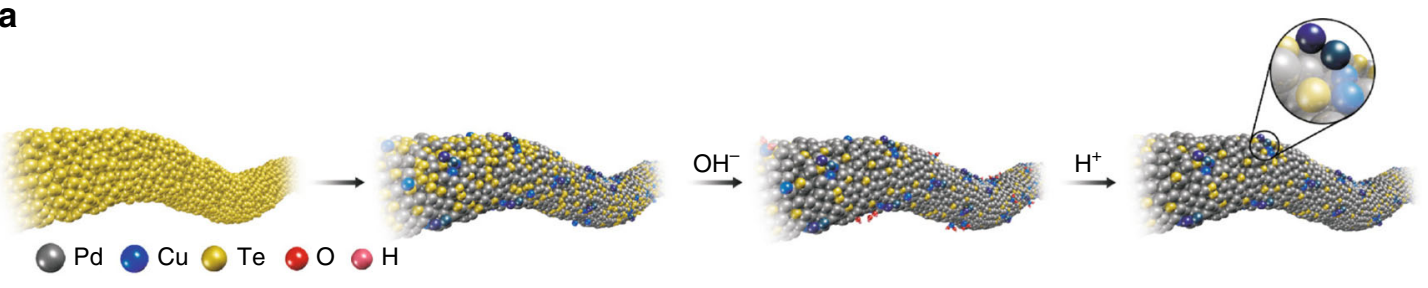

b

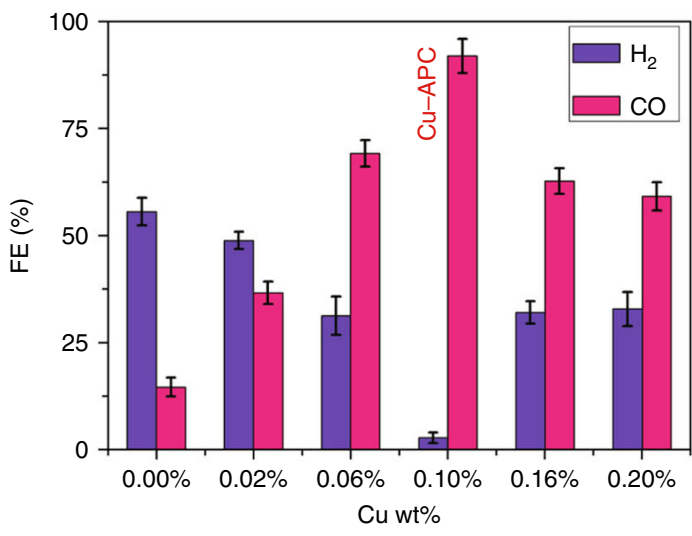

C
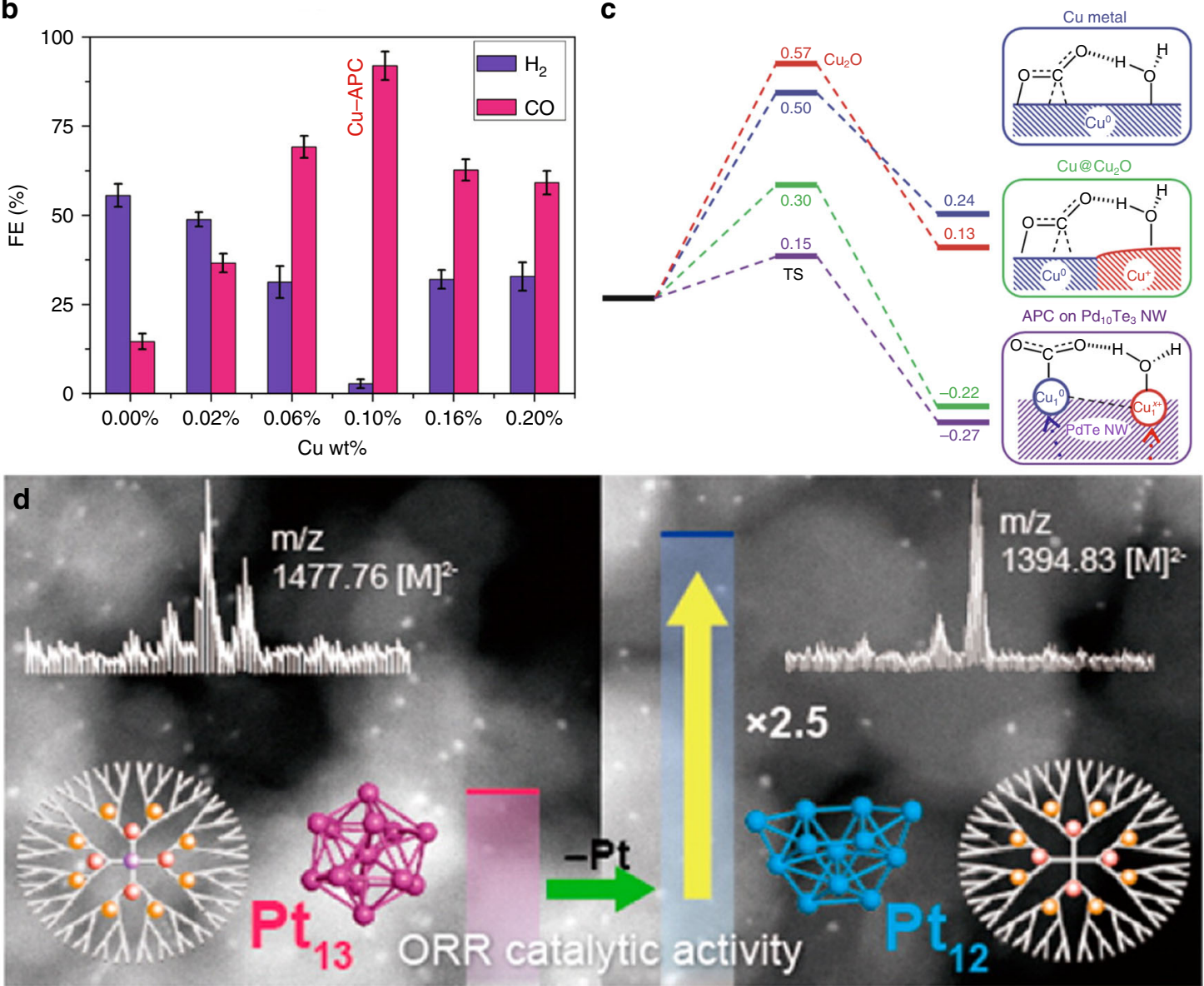

Fig. 6 The wet chemical reduction and dendrimer-based strategies. a Schematic illustration of the synthesis of atom-pair structured $\mathrm{Cu}$ anchored on $\mathrm{Pd}_{10} \mathrm{Te}_{3}$ nanowires. $\mathbf{b} \mathrm{FE}_{\mathrm{CO}}$ and $\mathrm{FE}_{\mathrm{H} 2}$ of samples with different $\mathrm{Cu}$ loading at $-0.78 \mathrm{~V}$ (versus $\mathrm{RHE}$ ) in $\mathrm{CO}_{2} \mathrm{RR}$. c Free energy profiles for $\mathrm{CO}_{2}$ activation on $\mathrm{Cu}, \mathrm{Cu} @ \mathrm{Cu}_{2} \mathrm{O}$, and $\mathrm{APC}$ of $\mathrm{Cu}_{1}{ }^{0}-\mathrm{Cu}_{1}{ }^{\mathrm{x}}+$ on $\mathrm{Pd}_{10} \mathrm{Te}_{3}$ nanowires. Reprinted by permission from Springer Nature Limited: Springer Nature, Nature Chemistry, ref. 30 (Jiao et al). Copyright (2019). d HAADF-STEM images and ORR catalytic activity of $\mathrm{Pt}_{13}$ (left) and $\mathrm{Pt}_{12}$ (right) clusters supported on mesoporous carbon. Reprinted by permission from ref. ${ }^{2}$. Copyright (2013) American Chemical Society.

materials 3,34 . Very recently, Sun et al. reported the synthesis of one-to-one $\mathrm{Pt}-\mathrm{Ru}$ dimers supported on $\mathrm{N}$-doped carbon nanotubes (NCNT) via a two-step ALD process (see Fig. 7a) ${ }^{34}$. They studied the depositions of Pt and Ru on NCNT, respectively, and found the easier deposition of Pt than Ru. So they firstly prepared isolated Pt atoms on the NCNT support by exposing NCNT with trimethyl(methylcyclopentadienyl)platinum(IV) ( $\left.\mathrm{MeCpPtMe}_{3}\right)$. Utilizing the different interactions between the Ru precursors and Pt or NCNT, they could deposit Ru atoms on Pt atoms selectively in the second step. In the AC-HAADF-STEM image, Pt atom was brighter than the Ru atom (see Fig. 7b). As depicted in the Fourier transforms of the Pt EXAFS spectra (see Fig. 7c), there was a main $\mathrm{Pt}-\mathrm{C} / \mathrm{N}$ bond peak $(1.6 \AA)$ and a small $\mathrm{Pt}-\mathrm{Ru}$ bond peak $(2.6 \AA)$ in the Pt-Ru dimer catalysts. Compared with the Pt SACs, the $\mathrm{Pt}-\mathrm{Ru}$ dimers exhibited better activity for electrochemical hydrogen evolution reaction (HER). Besides, the Pt-Ru dimers showed much higher mass activity (54 times) and excellent stability compared to commercial $\mathrm{Pt} / \mathrm{C}$ catalysts (see Fig. $7 \mathrm{~d}, \mathrm{e}$ ).
Based on the DFT calculations, when three $\mathrm{H}$ atoms adsorbed on both $\mathrm{Pt}$ and $\mathrm{Ru}$ sides of the $\mathrm{Pt}-\mathrm{Ru}$ dimer, the corresponding Gibbs free energy for $\mathrm{Pt}(3 \mathrm{H}) \mathrm{Ru}(3 \mathrm{H}) \rightarrow \mathrm{Pt}(3 \mathrm{H}) \mathrm{Ru}(2 \mathrm{H})$ was only $0.01 \mathrm{eV}$, which was smaller than that of Pt SACs $(0.1 \mathrm{eV})$ and Pt-Pt dimer catalyst $(-0.14 \mathrm{eV})$. In 2017, Lu et al. have used the same precursor to prepare Pt dimers on graphene by the ALD method. However, they proposed that the Pt dimers were in the oxidized form of $\mathrm{Pt}_{2} \mathrm{O}_{\mathrm{x}}{ }^{3}$.

In the works as mentioned earlier, the crucial factors in preparing isolated dimers are mainly two interactions: the steric hindrance between precursor molecules and the self-limiting reactions between precursor molecules and the supports. Therefore, the choice of the metallic precursor with huge ligands, such as methylcyclopentadienyl, is very fundamental. However, the high cost of these kinds of precursors hampers the practical application of the ALD method. All in all, this ALD method can prepare monometallic and bimetallic atomic clusters precisely, but the limited output in the sophisticated 
a

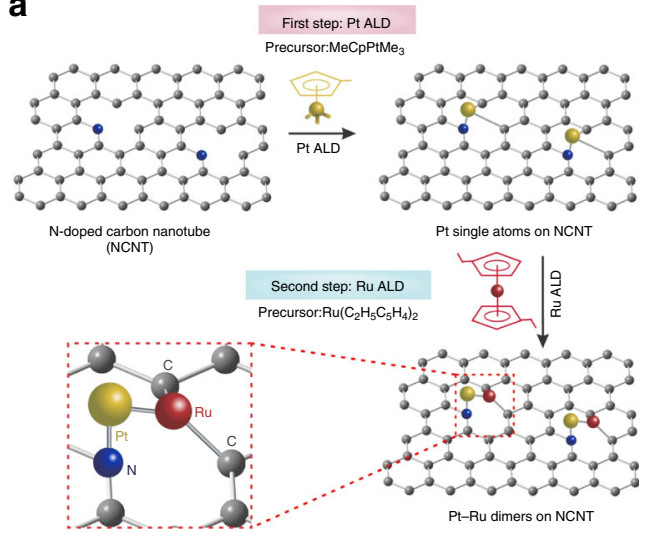

b

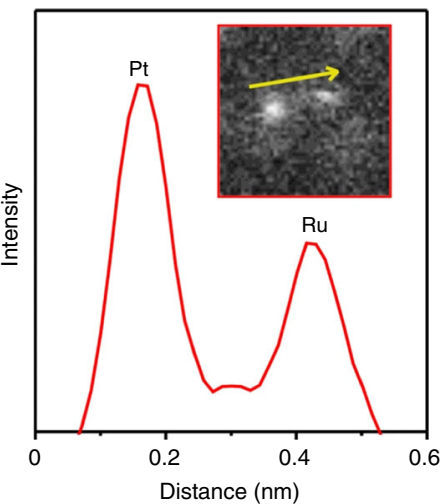

d

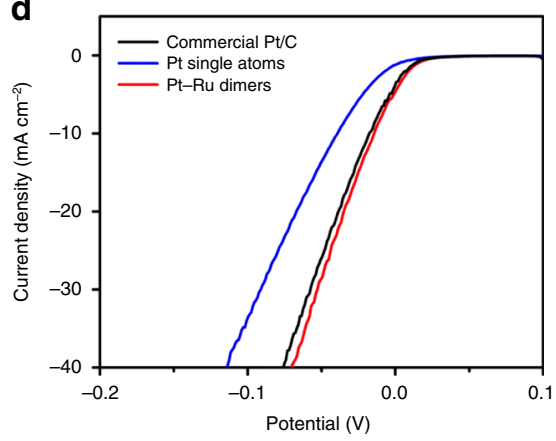

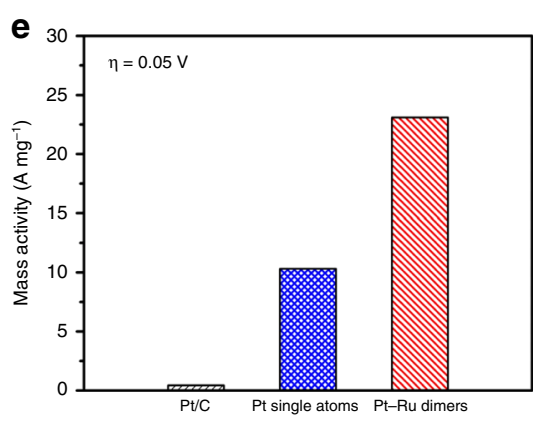

C

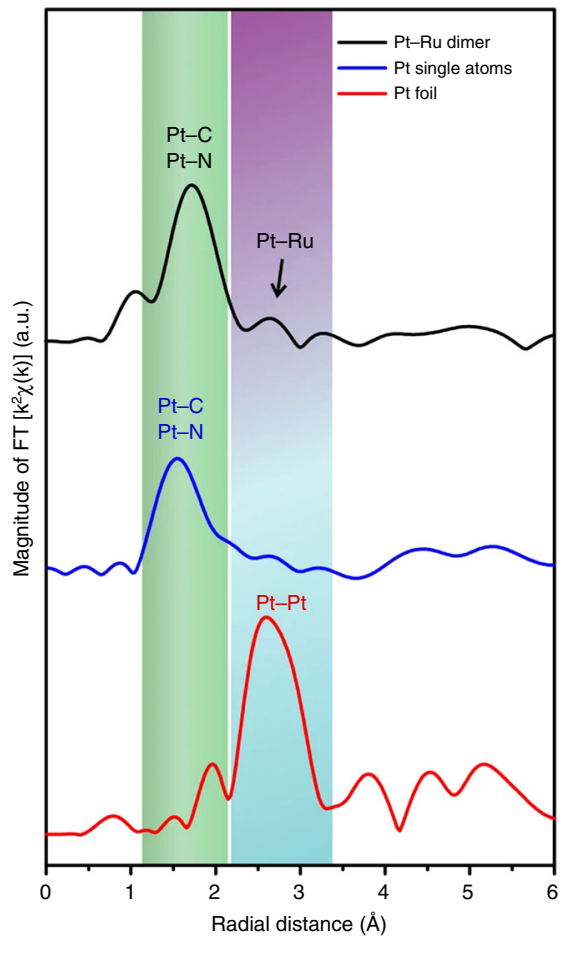

Fig. 7 The atomic layer deposition method. a Schematic illustration of the synthesis of Pt-Ru dimers on nitrogen-doped carbon nanotubes. $\mathbf{b}$ The intensity profile of one individual Pt-Ru dimer from the enlarged AC-HAADF-STEM image of inset. c Fourier transforms of the Pt EXAFS spectra for the Pt-Ru dimers, Pt single atoms, and Pt foil. d, e Electrocatalytic HER performance of Pt-Ru dimers and other samples. The polarization curves (d) and normalized mass activity at $0.05 \mathrm{~V}$ (e) of Pt-Ru dimers, Pt single atoms, and Pt/C catalysts ${ }^{34}$.

equipment and expensive precursors restrict the industrial scalability.

\section{Other methods}

Prof. Christopher et al. have developed strong electrostatic adsorption (SEA) method to deposit atomically dispersed heteroatom species consisting of late-transition metals on metallic oxides. Besides the weight loading, solution volume, and solution $\mathrm{pH}$, the precursors' choice is also significant. The surface oxygen species will change from hydroxyl $(\mathrm{OH})$ to oxygen anion $\left(\mathrm{O}^{-}\right)$ when the solution changes from neutral to alkaline ${ }^{98}$. By choosing a cationic precursor (always coordinated by four ammine molecules), the Coulombic attraction between the surface $\mathrm{O}^{-}$and the precursor promotes the deposition. Using this SEA method, they prepared $\mathrm{Pt}_{1} / \mathrm{CeO}_{2}{ }^{99}, \mathrm{Pt}_{1} / \mathrm{TiO}_{2}{ }^{100}$, and $\mathrm{Rh}-\mathrm{ReO}_{\mathrm{x}} / \mathrm{Al}_{2} \mathrm{O}_{3}{ }^{101}$ atomically dispersed catalysts.

Prof. Notestein et al. have developed the vapor-phase metalation by atomic layer deposition (ALD) in a metal-organic framework (MOF), during which the temperature is always in the range of $110-160^{\circ} \mathrm{C}$. The key to this strategy is the synthesis of NU-1000, a thermally stable, Zr-based MOF ${ }^{102}$. Various classes of volatile metal precursors containing alkyl, alkylamide, amidinate, carbonyl, and metallocene have been used to postmodify the NU$1000^{102-106}$. NU-1000 has 3 and $0.8 \mathrm{~nm}$ pores, and the deposited metal species mainly occupy the small pores centered between the nodes. Their loading is always smaller than eight metals/ $\mathrm{Zr}_{6}$ nodes.

Prof. Lopez-Quintela et al. have developed an electrochemical method to prepare metallic clusters with precise number ${ }^{107}$. Naked $\mathrm{Ag}_{2}$ and $\mathrm{Ag}_{3}$ clusters can be prepared using a very low concentration of $\mathrm{Ag}$ ions in solution ${ }^{108}$. Combing with DNAacting drugs, the $\mathrm{Ag}_{3}$ clusters that strongly intercalate with DNA play an essential role in improving cytotoxicity to human lung carcinoma cells ${ }^{109}$. Using a modified electrochemical method, $\mathrm{Au}_{3}, \mathrm{Au}_{5-7}$, and $\mathrm{Au}_{7-10}$ clusters were synthesized and supported on functionalized carbon nanotubes by a wet impregnation procedure. In the oxidation of thiophenol with $\mathrm{O}_{2}$, the single $\mathrm{Au}$ atoms are not active, while $\mathrm{Au}_{5-10}$ clusters are very active ${ }^{110}$.

\section{Outlook}

The development of synthesis methods is of great significance for the practical application of supported atomic clusters with welldefined structures. The synthesis of supported atomic clusters has made significant progress since early preparation in UHV conditions. In this perspective, six strategies have been summarized and classified for preparing supported atomic clusters, including gas-phase synthesis and size-selected strategy, precursorpreselected strategy, host-guest strategy, wet chemical reduction method, dendrimer-based strategy, and atomic layer deposition. In terms of precision synthesis, multilateral clusters, and industrial scalability, we have compared the advantages and disadvantages of each strategy and summarized them in Table 1.

We also highlight the catalyst application of supported atomic clusters and extend a discussion to the structure-activity relationship of them through the specific instances. With the indepth development of the researcher's cognition and the continuous progress of science and technology, the synthesis and application of supported atomic clusters in catalysis will be full of new opportunities but remain grand challenges.

\section{The synthesis of supported atomic clusters}

A critical restriction in the development of supported atomic clusters is the lack of general methods to efficiently and precisely access supported atomic clusters with high performance on a 
Table 1 Comparison of six synthesis strategies in terms of precision synthesis, multilateral clusters, and industrial scalability.

\begin{tabular}{l} 
Synthetic strategies \\
\hline Size-selected strategy \\
Precursor-preselected strategy \\
Host-guest strategy \\
Wet chemical reduction strategy \\
Dendrimer-based strategy \\
Atomic layer deposition strategy
\end{tabular}

large scale. Meanwhile, it is still challenging to achieve a controllable synthesis of supported atomic clusters with precise atom numbers. Up to now, most of the reported $\mathrm{M}_{2-10}$ supported atomic clusters have been prepared by chance. Thus, there is not systematic and practical guidance for the synthesis of supported atomic clusters. If we want to synthesize supported atomic clusters with precise atoms in a targeted way by chemical methods, we had better have a deeper understanding of the critical factors to obtain stable structures. For example, the competitive binding between metal-support and metal-metal determines the stability of corresponding SACs. When the former is thermodynamically stable than the latter, it may be a driving force for the transformation of SAC from nanoparticles. Whether the experimental conditions can provide enough energy for the kinetic barrier of the transformation is another crucial facet. Therefore, employing systematic experiments and theoretical calculations, we may be able to predict the more stable configuration of single-cluster catalysts anchored on different supports, which is crucial for the synthesis of supported atomic clusters.

To develop the synthesis of supported metallic atomic clusters, following six factor can be considered. (1) The bottom-up strategy to prepare supported atomic clusters should be given enough attention, during which the monoatomic precursors are absorbed, assembled, reduced, and confined by the defects of the supports. (2) The recently developed matrix assembly cluster source (MACS) ${ }^{111}$ and high-power impulse magnetron sputtering (HiPIMS) ${ }^{112}$ methods present a bridge between the laboratory research scale and the industrial levels. The former method produces cluster-decorated powder catalysts at the gram scale. (3) The production rates of simple nanoparticles in conventional aerosol flame technology reach $25 \mathrm{t} / \mathrm{h}$, so it may be extended to produce supported atomic clusters in large scale, during which the main challenge is the precise control of the atomicity ${ }^{113}$. (4) The top-down strategy, based on the decomposing of ordered nanostructures into smaller species, is worth a test, which is proven effective in the scalable synthesis of SACs. (5) The strategy for precise handling of the nuclearity of supported atomic clusters by the wet chemical method should be developed to enrich the variety and obtain multifunctional materials. (6) The manipulation of the electronic structures of supported atomic clusters may be achieved by tuning the geometric structure of atomic clusters, or by changing the chemical environment of the supports.

\section{The characterization of supported atomic clusters}

As we know, AC-HAADF-STEM analysis usually represents the direct two-dimensional projection of the supported atomic clusters samples along the direction of the incident beam. Thus, the observed atomic structure images cannot fully reflect the threedimensional perspective of the supported atomic clusters samples. Besides, the thickness of an as-prepared catalyst in the $z$-axis is always tens or hundreds of nanometers, which may make several single-cluster sites coincide in the direction of the incident beam. In particular, when the supported atomic clusters contain multiple metal atoms with similar ordinal numbers, such as iron, cobalt, and nickel, they will display similar contrast in HAADFSTEM images, which will make it more difficult to identify them from each other. Therefore, there are many difficulties in the unambiguous characterization of supported atomic clusters.

The X-ray absorption fine spectrum (XAFS) is a powerful tool to analyze the chemical environment and coordination structure of the supported atomic clusters. It is not like the SACs system that no metal-metal bond can strongly prove the atomic dispersion of metal species. For the supported atomic clusters, it is not only necessary to confirm that there are no nanoparticles, but also to prove that there are limited metal-metal bonds among metal cluster atoms. Also, the single clusters supported on host materials doped by various kinds of light atoms $(\mathrm{C}, \mathrm{O}, \mathrm{N}, \mathrm{P}, \mathrm{S}$, etc.), the atomic structures of the supported atomic clusters are too complex to be determined, owing to a variety of possible structural coordination between metals and light atoms. Notably, the identification of dimers and trimers supported on the same material is much more difficult. To make the characterization easier, researchers can employ ultrathin or 2D supports for preparing the supported atomic clusters. With the advances of characterization, the $3 \mathrm{D}$ reconstruction technique may provide a possible way to confirm the more stereoscopic or realistic atom structure for supported atomic clusters. Combining with the theoretical calculation, we can provide an effective way for the investigation of supported atomic clusters.

\section{The catalytic study of supported atomic clusters}

The development of a synthesis strategy also promotes the investigation of catalytic systems under actual reaction conditions. In the reported papers, the performances of SACs are sometimes better than these of the supported atomic clusters, on the contrary, it often appears, and there is a lack of disciplinary and systematic understanding of supported atomic clusters in catalysis. The essential reason is derived from the uncontrollable design in synthesis. For example, the system of $\mathrm{Pd}_{1}$ and $\mathrm{Pd}_{6}$ reported in the literature is also an unexpected finding. We can attempt to design and synthesize the realistic $\mathrm{Pd}_{1-10}$ materials and systematically study their differences in structure and performance, which will provide an experimental basis for the study of real active species and the design of optimized catalysts. In addition, the strong interaction between metals and supports affects the geometry and stability of the supported atomic clusters, owing to the charge transfer between supports and clusters. Therefore, it is one of the essential factors in the study of the catalytic applications of supported atomic clusters.

Five aspects should also be given enough attention. (1) The dynamic behavior of the supported atomic clusters during catalytic processes. In situ spectroscopic (X-ray absorption spectroscopy, XPS, X-ray diffraction) and microscopic (TEM) techniques that allow tracking the transformation during the catalytic process will be particularly helpful. (2) The non-uniformity of the support materials denotes that not all the single-cluster centers in 
supported atomic clusters are equally active. This non-uniformity may bring about the diversity of the catalytic performances, including the activity, selectivity, and stability. (3) Heterogeneous supported atomic clusters can catalyze the homogeneous reactions with distinct yield and selectivity. (4) Based on the scalable synthesis of supported atomic clusters, we should promote the application of supported atomic clusters in the industrial field. (5) Concomitant advances in synthesis, characterization, and development in molecular modeling, have been beneficial in understanding the structure-function relationships of supported atomic clusters, as well as to predict their catalytic performance. For a target reaction, a specific catalyst will be designed at the atomic and molecular scales, and the green, atomic economy catalytic processes will be realized.

Received: 3 December 2019; Accepted: 15 October 2020;

Published online: 18 November 2020

\section{References}

1. Herzing, A. A., Kiely, C. J., Carley, A. F., Landon, P. \& Hutchings, G. J. Identification of active gold nanoclusters on iron oxide supports for $\mathrm{CO}$ oxidation. Science 321, 1331-1335 (2008).

2. Imaoka, T. et al. Magic number $\mathrm{Pt}_{13}$ and misshapen $\mathrm{Pt}_{12}$ clusters: which one is the better catalyst? J. Am. Chem. Soc. 135, 13089-13095 (2013).

3. Yan, H. et al. Bottom-up precise synthesis of stable platinum dimers on graphene. Nat. Commun. 8, 1070 (2017). This paper reports bottom-up synthesis of stable $\mathrm{Pt}_{2}$ dimers on graphene by atomic layer deposition.

4. Vorobyeva, E. et al. Atom-by-atom resolution of structure-function relations over low-nuclearity metal catalysts. Angew. Chem. Int. Ed. 58, 8724-8729 (2019). This paper reveals the different nuclearity impacts of $P d x(x=1,2$, 3) in semi-hydrogenation of alkynes and Suzuki coupling.

5. Baxter, E. T., Ha, M.-A., Cass, A. C., Alexandrova, A. N. \& Anderson, S. L. Ethylene dehydrogenation on $\mathrm{Pt}_{4,7,8}$ clusters on $\mathrm{Al}_{2} \mathrm{O}_{3}$ : strong cluster size dependence linked to preferred catalyst morphologies. ACS Catal. 7, 3322-3335 (2017).

6. Li, G. et al. Sn-modification of $\mathrm{Pt}_{7} /$ alumina model catalysts: suppression of carbon deposition and enhanced thermal stability. J. Chem. Phys. 152, 024702-024702 (2020).

7. Kaden, W. E., Wu, T., Kunkel, W. A. \& Anderson, S. L. Electronic structure controls reactivity of size-selected Pd clusters adsorbed on $\mathrm{TiO}_{2}$ surfaces. Science 326, 826-829 (2009).

8. Watanabe, Y., Wu, X., Hirata, H. \& Isomura, N. Size-dependent catalytic activity and geometries of size-selected Pt clusters on $\mathrm{TiO}_{2}(110)$ surfaces. Catal. Sci. Technol. 1, 1490-1495 (2011).

9. Isomura, $\mathrm{N}$., $\mathrm{Wu}, \mathrm{X}$. \& Watanabe, Y. Atomic-resolution imaging of sizeselected platinum clusters on $\mathrm{TiO}_{2}(110)$ surfaces. J. Chem. Phys. 131, 164707 (2009).

10. Sun, T., Xu, L., Wang, D. \& Li, Y. Metal organic frameworks derived single atom catalysts for electrocatalytic energy conversion. Nano Res. 12, 2067-2080 (2019).

11. Ye, X. et al. Insight of the stability and activity of platinum single atoms on ceria. Nano Res. 12, 1401-1409 (2019).

12. Mitchell, S., Vorobyeva, E. \& Pérez-Ramírez, J. The multifaceted reactivity of single-atom heterogeneous catalysts. Angew. Chem. Int. Ed. 57, 15316-15329 (2018).

13. Li, X., Rong, H., Zhang, J., Wang, D. \& Li, Y. Modulating the local coordination environment of single-atom catalysts for enhanced catalytic performance. Nano Res. 13, 1842-1855 (2020).

14. Zhuang, Z., Kang, Q., Wang, D. \& Li, Y. Single-atom catalysis enables longlife, high-energy lithium-sulfur batteries. Nano Res. 13, 1856-1866 (2020).

15. Xu, Q. et al. Coordination structure dominated performance of single-atomic Pt catalyst for anti-Markovnikov hydroboration of alkenes. Sci. China Mater. 63, 972-981 (2020).

16. Wang, H. et al. Surpassing the single-atom catalytic activity limit through paired Pt-O-Pt ensemble built from isolated Pt1 atoms. Nat. Commun. 10, 3808 (2019).

17. Zhang, B.-W. et al. New monatomic layer clusters for advanced catalysis materials. Sci. China-Mater. 62, 149-153 (2019).

18. Ji, S. et al. Confined pyrolysis within metal-organic frameworks to form uniform $\mathrm{Ru}_{3}$ clusters for efficient oxidation of alcohols. J. Am. Chem. Soc. 139, 9795-9798 (2017). This paper illustrates the confined effect of zeolitic imidazolate frameworks in the synthesis of Ru3 atomic clusters by precursor preselected strategy.

19. Wang, J. et al. Design of N-coordinated dual-metal sites: a stable and active Ptfree catalyst for acidic oxygen reduction reaction. J. Am. Chem. Soc. 139, 17281-17284 (2017).

20. Lee, S. S., Fan, C. Y., Wu, T. P. \& Anderson, S. L. CO oxidation on Aun/TiO catalysts produced by size-selected cluster deposition. J. Am. Chem. Soc. 126, 5682-5683 (2004).

21. Yoon, B. et al. Charging effects on bonding and catalyzed oxidation of $\mathrm{CO}$ on $\mathrm{Au}_{8}$ clusters on MgO. Science 307, 403-407 (2005).

22. Vajda, S. et al. Subnanometre platinum clusters as highly active and selective catalysts for the oxidative dehydrogenation of propane. Nat. Mater. 8, 213-216 (2009). This paper displays the high activity and selectivity of sizeselected Pt8-10 atomic clusters towards formation of propylene in the dehydrogenation of propane.

23. Lei, Y. et al. Increased silver activity for direct propylene epoxidation via subnanometer size effects. Science 328, 224-228 (2010).

24. Kwon, G. et al. Size-dependent subnanometer Pd cluster $\left(\mathrm{Pd}_{4}, \mathrm{Pd}_{6}\right.$, and $\left.\mathrm{Pd}_{17}\right)$ water oxidation electrocatalysis. ACS Nano 7, 5808-5817 (2013).

25. Crampton, A. S. et al. Structure sensitivity in the nonscalable regime explored via catalysed ethylene hydrogenation on supported platinum nanoclusters. Nat. Commun. 7, 10389 (2016).

26. Anderson, J. R. \& Howe, R. F. Generation of a supported iridium catalyst of extremely high dispersion. Nature 268, 129-130 (1977).

27. Tian, $\mathrm{S}$. et al. Carbon nitride supported $\mathrm{Fe}_{2}$ cluster catalysts with superior performance for alkene epoxidation. Nat. Commun. 9, 2353 (2018).

28. Ren, W. et al. Isolated diatomic Ni-Fe metal-nitrogen sites for synergistic electroreduction of $\mathrm{CO}_{2}$. Angew. Chem. Int. Ed. 58, 6972-6976 (2019).

29. Han, X. et al. Atomically dispersed binary Co-Ni sites in nitrogen-doped hollow carbon nanocubes for reversible oxygen reduction and evolution. Adv Mater. 31, 1905622 (2019).

30. Jiao, J. et al. Copper atom-pair catalyst anchored on alloy nanowires for selective and efficient electrochemical reduction of $\mathrm{CO}_{2}$. Nat. Chem. 11, 222-228 (2019). This paper introduces an electrocatalyst featuring two adjacent $\mathrm{Cu}$ atoms on $\mathrm{Pd}_{10} \mathrm{Te}_{3}$ nanowires, can selectively transform $\mathrm{CO}_{2}$ to CO.

31. Dai, S. et al. Platinum-trimer decorated cobalt-palladium core-shell nanocatalyst with promising performance for oxygen reduction reaction. Nat. Commun. 10, 440 (2019).

32. Zheng, J. \& Dickson, R. M. Individual water-soluble dendrimer-encapsulated silver nanodot fluorescence. J. Am. Chem. Soc. 124, 13982-13983 (2002).

33. Yamamoto, K., Imaoka, T., Tanabe, M. \& Kambe, T. New horizon of nanoparticle and cluster catalysis with dendrimers. Chem. Rev. 120, 1397-1437 (2019). This paper reviews the synthesis and catalytic performances of dendrimer-encapsulated metal nanoparticles and clusters.

34. Zhang, L. et al. Atomic layer deposited Pt-Ru dual-metal dimers and identifying their active sites for hydrogen evolution reaction. Nat. Commun. 10, 4936 (2019).

35. Mao, B.-H. et al. Oxidation and reduction of size-selected subnanometer Pd clusters on $\mathrm{Al}_{2} \mathrm{O}_{3}$ surface. J. Chem. Phys. 138, 214304 (2013).

36. Benz, L. et al. Landing of size-selected Agn + clusters on single crystal $\mathrm{TiO}_{2}$ (110)-(1x1) surfaces at room temperature. J. Chem. Phys. 122, 81102 (2005).

37. Tyo, E. C. \& Vajda, S. Catalysis by clusters with precise numbers of atoms. Nat. Nanotechnol. 10, 577 (2015). This paper reviews the synthesis, characterization, and the catalytic properties of size-selected supported clusters.

38. Zandkarimi, B. \& Alexandrova, A. N. Dynamics of subnanometer Pt clusters can break the scaling relationships in catalysis. J. Phys. Chem. Lett. 10, 460-467 (2019)

39. Chen, Z. W., Yan, J.-M. \& Jiang, Q. Single or double: which is the altar of atomic catalysts for nitrogen reduction reaction? Small Methods 3, 1800291 (2019).

40. Cheng, C., Zhang, X. L., Yang, Z. X. \& Zhou, Z. $\mathrm{Cu}_{3}$-cluster-doped monolayer $\mathrm{Mo}_{2} \mathrm{CO}_{2}$ (MXene) as an electron reservoir for catalyzing a $\mathrm{CO}$ oxidation reaction. ACS Appl. Mater. Interfaces 10, 32903-32912 (2018).

41. Zhang, X., Chen, A., Zhang, Z. \& Zhou, Z. Double-atom catalysts: transition metal dimer-anchored $\mathrm{C} 2 \mathrm{~N}$ monolayers as $\mathrm{N} 2$ fixation electrocatalysts. $J$. Mater. Chem. A 6, 18599-18604 (2018).

42. Liu, J.-C. et al. Heterogeneous $\mathrm{Fe}_{3}$ single-cluster catalyst for ammonia synthesis via an associative mechanism. Nat. Commun. 9, 1610 (2018)

43. Ma, X.-L., Liu, J.-C., Xiao, H. \& Li, J. Surface single-cluster catalyst for $\mathrm{N}_{2}$-to$\mathrm{NH}_{3}$ thermal conversion. J. Am. Chem. Soc. 140, 46-49 (2018).

44. Winans, R. E. et al. Reactivity of supported platinum nanoclusters studied by in situ GISAXS: clusters stability under hydrogen. Top. Catal. 39, 145-149 (2006).

45. Schmidt, M., Kusche, R., von Issendorff, B. \& Haberland, H. Irregular variations in the melting point of size-selected atomic clusters. Nature 393, 238-240 (1998). 
46. Fayet, P. \& Woste, L. Production and study of metal cluster ions. Surf. Sci. 156, 134-139 (1985).

47. Gantefor, G., Siekmann, H. R., Lutz, H. O. \& Meiwesbroer, K. H. Pure metal and metal-doped rare-gas clusters grown in a pulsed-arc cluster ion-source. Chem. Phys. Lett. 165, 293-296 (1990).

48. $\mathrm{Li}, \mathrm{A}$. et al. Using ambient ion beams to write nanostructured patterns for surface enhanced raman spectroscopy. Angew. Chem. Int. Ed. 53, 12528-12531 (2014).

49. Methling, R. P. et al. Magnetic studies on mass-selected iron particles. Eur. Phys. J. D. 16, 173-176 (2001).

50. Miller, P. E. \& Denton, M. B. The quadrupole mass filter-basic operating concepts. J. Chem. Educ. 63, 617-622 (1986).

51. Oliver-Meseguer, J., Cabrero-Antonino, J. R., Dominguez, I., Leyva-Perez, A. \& Corma, A. Small gold clusters formed in solution give reaction turnover numbers of 107 at room temperature. Science 338, 1452-1455 (2012).

52. Bromann, K. et al. Controlled deposition of size-selected silver nanoclusters. Science 274, 956-958 (1996).

53. Bals, S. et al. Atomic scale dynamics of ultrasmall germanium clusters. Nat. Commun. 3, 897 (2012).

54. Nonose, S., Sone, Y., Onodera, K., Sudo, S. \& Kaya, K. Structure and reactivity of bimetallic cobalt-vanadium (ConVm) clusters. J. Phys. Chem. 94, 2744-2746 (1990).

55. Popok, V. N., Barke, I., Campbell, E. E. B. \& Meiwes-Broer, K.-H. Clustersurface interaction: from soft landing to implantation. Surf. Sci. Rep. 66, 347-377 (2011).

56. Deheer, W. A. The physics of simple metal-clusters-experimental aspects and simple-models. Rev. Mod. Phys. 65, 611-676 (1993).

57. Chen, T., Yao, Q., Nasaruddin, R. R. \& Xie, J. Electrospray ionization mass spectrometry: a powerful platform for noble-metal nanocluster analysis. Angew. Chem. Int. Ed. 58, 11967-11977 (2019).

58. Guo, L.-W. et al. Contributions of distinct gold species to catalytic reactivity for carbon monoxide oxidation. Nat. Commun. 7, 13481 (2016).

59. Valden, M., Lai, X. \& Goodman, D. W. Onset of catalytic activity of gold clusters on titania with the appearance of nonmetallic properties. Science 281, 1647-1650 (1998).

60. Heiz, U., Sanchez, A., Abbet, S. \& Schneider, W. D. Catalytic oxidation of carbon monoxide on monodispersed platinum clusters: each atom counts. J. Am. Chem. Soc. 121, 3214-3217 (1999).

61. Bonanni, S., Ait-Mansour, K., Harbich, W. \& Brune, H. Effect of the $\mathrm{TiO}_{2}$ reduction state on the catalytic $\mathrm{CO}$ oxidation on deposited size-selected $\mathrm{Pt}$ clusters. J. Am. Chem. Soc. 134, 3445-3450 (2012).

62. Moseler, M. et al. Oxidation state and symmetry of magnesia-supported $\mathrm{Pd}_{13} \mathrm{O}_{\mathrm{x}}$ nanocatalysts influence activation barriers of $\mathrm{CO}$ oxidation. J. Am. Chem. Soc. 134, 7690-7699 (2012).

63. Su, Y.-Q., Filot, I. A. W., Liu, J.-X. \& Hensen, E. J. M. Stable Pd-doped ceria structures for $\mathrm{CH}_{4}$ activation and CO oxidation. ACS Catal. 8, 75-80 (2018).

64. Liu, J. et al. Preparation, characterization and origin of highly active and thermally stable $\mathrm{Pd}-\mathrm{Ce}_{0.8} \mathrm{Zr}_{0.2} \mathrm{O}_{2}$ catalysts via sol-evaporation induced selfassembly method. Environ. Sci. Technol. 48, 12403-12410 (2014).

65. Gulyaev, R. V. et al. Highly active PdCeOx composite catalysts for lowtemperature $\mathrm{CO}$ oxidation, prepared by plasma-arc synthesis. Appl. Catal. BEnviron. 147, 132-143 (2014).

66. Negreiros, F. R. et al. Bimetallic Ag-Pt sub-nanometer supported clusters as highly efficient and robust oxidation catalysts. Angew. Chem. Int. Ed. 57, 1209-1213 (2018).

67. Yin, C. R. et al. Alumina-supported sub-nanometer Pt10 clusters: amorphization and role of the support material in a highly active CO oxidation catalyst. J. Mater. Chem. A 5, 4923-4931 (2017).

68. Lee, S. et al. Selective propene epoxidation on immobilized $A_{u_{6-10}}$ clusters: the effect of hydrogen and water on activity and selectivity. Angew. Chem. Int. Ed. 48, 1467-1471 (2009).

69. Vorobyeva, E. et al. Tailoring the framework composition of carbon nitride to improve the catalytic efficiency of the stabilized palladium atoms. J. Mater. Chem. A 5, 16393-16403 (2017).

70. Chen, Z. et al. Single-atom heterogeneous catalysts based on distinct carbon nitride scaffolds. Natl Sci. Rev. 5, 642-652 (2018)

71. Lu, J., Serna, P., Aydin, C., Browning, N. D. \& Gates, B. C. Supported molecular iridium catalysts: Resolving effects of metal nuclearity and supports as ligands. J. Am. Chem. Soc. 133, 16186-16195 (2011).

72. Argo, A. M., Odzak, J. F., Lai, F. S. \& Gates, B. C. Observation of ligand effects during alkene hydrogenation catalyzed by supported metal clusters. Nature 415, 623-626 (2002).

73. von Weber, A. \& Anderson, S. L. Electrocatalysis by mass-selected Ptn clusters. Acc. Chem. Res. 49, 2632-2639 (2016).

74. Vajda, S. \& White, M. G. Catalysis applications of size-selected cluster deposition. ACS Catal. 5, 7152-7176 (2015).

75. Lee, S. et al. Subnanometer cobalt oxide clusters as selective low temperature oxidative dehydrogenation catalysts. Nat. Commun. 10, 954 (2019).
76. Anderson, J. R. \& Mainwaring, D. E. Use of a bimetallic molecular cluster compound for the preparation of a dispersed bimetallic catalyst: methylcyclopentane hydrogenolysis. J. Catal. 35, 162-165 (1974).

77. Smith, G. C., Chojnacki, T. P., Dasgupta, S. R., Iwatate, K. \& Watters, K. L. Surface-supported metal cluster carbonyls. I. Decarbonylation and aggregation reactions of rhodium clusters on alumina. Inorg. Chem. 14, 1419-1421 (1975)

78. Plummer, E. W., Salaneck, W. R. \& Miller, J. S. Photoelectron spectra of transition-metal carbonyl complexes: comparison with the spectra of adsorbed CO. Phys. Rev. B 18, 1673-1701 (1978).

79. Tanaka, K., Watters, K. L. \& Howe, R. F. Characterization of supported iridium catalysts prepared from $\operatorname{Ir}_{4}(\mathrm{CO})_{12}$. J. Catal. 75, 23-38 (1982).

80. Légaré, P., Sakisaka, Y., Brucker, C. F. \& Rhodin, T. N. Electronic structure of highly dispersed supported transition metal clusters. Surf. Sci. 139, 316-332 (1984).

81. Anderson, J. R., Elmes, P. S., Howe, R. F. \& Mainwaring, D. E. Preparation of some supported metallic catalysts from metallic cluster carbonyls. J. Catal. 50, 508-518 (1977).

82. $\mathrm{Xu}, \mathrm{Z}$. et al. Size-dependent catalytic activity of supported metal clusters. Nature 372, 346-348 (1994).

83. Ji, S. et al. Atomically dispersed ruthenium species inside metal-organic frameworks: combining the high activity of atomic sites and the molecular sieving effect of MOFs. Angew. Chem. Int. Ed. 58, 4271-4275 (2019).

84. Xin, P. et al. Revealing the active species for aerobic alcohol oxidation by using uniform supported palladium catalysts. Angew. Chem. Int. Ed. 57, 4642-4646 (2018). This paper points out the lower catalytic activity of $\mathrm{Pd6} / \mathrm{CeO} 2$ than $\mathrm{Pd1} / \mathrm{CeO2}$ in the oxidation reaction of aerobic alcohol.

85. Fortea-Pérez, F. R. et al. The MOF-driven synthesis of supported palladium clusters with catalytic activity for carbene-mediated chemistry. Nat. Mater. 16, 760 (2017). This paper highlights the synthesis of a MOF-supported Pd4 clusters and its catalytic performances in carbene-mediated reactions of diazoacetates.

86. Mon, M. et al. Synthesis of densely packaged, ultrasmall Pt02 clusters within a thioether-functionalized MOF: catalytic activity in industrial reactions at low temperature. Angew. Chem. Int. Ed. 57, 6186-6191 (2018).

87. Wang, J. et al. N-coordinated dual-metal single-site catalyst for lowtemperature CO oxidation. ACS Catal. 10, 2754-2761 (2020).

88. Xiao, M. et al. Climbing the apex of the ORR volcano plot via binuclear site construction: electronic and geometric engineering. J. Am. Chem. Soc. 141, 17763-17770 (2019).

89. Zhao, J., Zhao, J. X., Li, F. Y. \& Chen, Z. F. Copper dimer supported on a $\mathrm{C}_{2} \mathrm{~N}$ layer as an efficient electrocatalyst for $\mathrm{CO}_{2}$ reduction reaction: a computational study. J. Phys. Chem. C. 122, 19712-19721 (2018).

90. Zheng, J., Petty, J. T. \& Dickson, R. M. High quantum yield blue emission from water-soluble Au8 nanodots. J. Am. Chem. Soc. 125, 7780-7781 (2003)

91. Zheng, J., Zhang, C. \& Dickson, R. M. Highly fluorescent, water-soluble, sizetunable gold quantum dots. Phys. Rev. Lett. 93, 077402 (2004).

92. Yamamoto, K. et al. Size-specific catalytic activity of platinum clusters enhances oxygen reduction reactions. Nat. Chem. 1, 397-402 (2009).

93. Kambe, T., Haruta, N., Imaoka, T. \& Yamamoto, K. Solution-phase synthesis of $\mathrm{Al}_{13}{ }^{-}$using a dendrimer template. Nat. Commun. 8, 2046 (2017).

94. Maeno, Z., Mitsudome, T., Mizugaki, T., Jitsukawa, K. \& Kaneda, K. Selective synthesis of Rh5 carbonyl clusters within a polyamine dendrimer for chemoselective reduction of nitro aromatics. Chem. Commun. 50, 6526-6529 (2014).

95. Tsukamoto, T., Kambe, T., Nakao, A., Imaoka, T. \& Yamamoto, K. Atomhybridization for synthesis of polymetallic clusters. Nat. Commun. 9, 3873 (2018).

96. Yamamoto, K. \& Imaoka, T. Precision synthesis of subnanoparticles using dendrimers as a superatom synthesizer. Acc. Chem. Res. 47, 1127-1136 (2014).

97. Imaoka, T., Kitazawa, H., Chun, W.-J. \& Yamamoto, K. Finding the most catalytically active platinum clusters with low atomicity. Angew. Chem. Int. Ed. 54, 9810-9815 (2015).

98. DeRita, L. et al. Catalyst architecture for stable single atom dispersion enables site-specific spectroscopic and reactivity measurements of $\mathrm{CO}$ adsorbed to $\mathrm{Pt}$ atoms, oxidized Pt clusters, and metallic Pt clusters on $\mathrm{TiO}_{2}$. J. Am. Chem. Soc 139, 14150-14165 (2017)

99. Resasco, J. et al. Uniformity is key in defining structure-function relationships for atomically dispersed metal catalysts: The case of $\mathrm{Pt} / \mathrm{CeO}_{2}$. J. Am. Chem. Soc. 142, 169-184 (2020).

100. DeRita, L. et al. Structural evolution of atomically dispersed Pt catalysts dictates reactivity. Nat. Mater. 18, 746-751 (2019).

101. Ro, I., Xu, M., Graham, G. W., Pan, X. \& Christopher, P. Synthesis of heteroatom $\mathrm{Rh}-\mathrm{ReOx}$ atomically dispersed species on $\mathrm{Al}_{2} \mathrm{O}_{3}$ and their tunable catalytic reactivity in ethylene hydroformylation. ACS Catal. 9, 10899-10912 (2019).

102. Mondloch, J. E. et al. Vapor-phase metalation by atomic layer deposition in a metal-organic framework. J. Am. Chem. Soc. 135, 10294-10297 (2013). 
103. Kim, I. S. et al. Sinter-resistant platinum catalyst supported by metal-organic framework. Angew. Chem. Int. Ed. 57, 909-913 (2018).

104. Gallington, L. C. et al. Regioselective atomic layer deposition in metal-organic frameworks directed by dispersion interactions. J. Am. Chem. Soc. 138, 13513-13516 (2016).

105. Li, Z. et al. Sintering-resistant single-site nickel catalyst supported by metal-organic framework. J. Am. Chem. Soc. 138, 1977-1982 (2016).

106. Kim, I. S. et al. The synthesis science of targeted vapor-phase metal-organic framework postmodification. J. Am. Chem. Soc. 142, 242-250 (2020).

107. Santiago Gonzalez, B. et al. One step synthesis of the smallest photo luminescent and paramagnetic PVP-protected gold atomic clusters. Nano Lett. 10, 4217-4221 (2010).

108. Buceta, D. et al. $\mathrm{Ag}_{2}$ and $\mathrm{Ag}_{3}$ clusters: Synthesis, characterization, and interaction with DNA. Angew. Chem. Int. Ed. 54, 7612-7616 (2015).

109. Porto, V. et al. Silver atomic quantum clusters of three atoms for cancer therapy: targeting chromatin compaction to increase the therapeutic index of chemotherapy. Adv. Mater. 30, 1801317 (2018).

110. Corma, A. et al. Exceptional oxidation activity with size-controlled supported gold clusters of low atomicity. Nat. Chem. 5, 775-781 (2013).

111. Palmer, R. E., Cai, R. \& Vernieres, J. Synthesis without solvents: the cluster (nanoparticle) beam route to catalysts and sensors. Acc. Chem. Res. 51, 2296-2304 (2018).

112. Zhang, C. et al. Advanced nanocluster ion source based on highpower impulse magnetron sputtering and time-resolved measurements of nanocluster formation. J. Phys. Chem. A 117, 10211-10217 (2013).

113. Zhao, X. et al. Engineering $\mathrm{TiO}_{2}$ supported $\mathrm{Pt}$ sub-nanoclusters via introducing variable valence $\mathrm{Co}$ ion in high-temperature flame for $\mathrm{CO}$ oxidation. Nanoscale 10, 13384-13392 (2018).

\section{Acknowledgements}

This work was supported by the National Key R\&D Program of China (2018YFA0702003, 2016YFA0202801), the National Natural Science Foundation of China (51631001, 51872030, 51702016, 51902023, 21801015, 21890383, 21671117, and 21871159), Science and Technology Key Project of Guangdong Province of China (2020B010188002), Beijing Municipal Science \& Technology Commission No.

Z191100007219003, and the Beijing Institute of Technology Research Fund Program for
Young Scholars. Prof. Jie Zhao, East China University of Science and Technology, for help with graphing. We thank Dr. Yu Mao for assistance on polishing the manuscript.

\section{Author contributions}

H.R. and S.J. contributed in developing the concept, writing, preparing the images, and gathering and analyzing the reported data. J.Z. and Y.L. contributed to the discussion of the literature. D.W. has the major role of developing the idea and leading the work.

\section{Competing interests}

The authors declare no competing interests.

\section{Additional information}

Correspondence and requests for materials should be addressed to S.J., J.Z. or D.W.

Peer review information Nature Communications thanks the anonymous reviewer(s) for their contribution to the peer review of this work.

Reprints and permission information is available at http://www.nature.com/reprints

Publisher's note Springer Nature remains neutral with regard to jurisdictional claims in published maps and institutional affiliations.

(c) (i) Open Access This article is licensed under a Creative Commons Attribution 4.0 International License, which permits use, sharing, adaptation, distribution and reproduction in any medium or format, as long as you give appropriate credit to the original author(s) and the source, provide a link to the Creative Commons license, and indicate if changes were made. The images or other third party material in this article are included in the article's Creative Commons license, unless indicated otherwise in a credit line to the material. If material is not included in the article's Creative Commons license and your intended use is not permitted by statutory regulation or exceeds the permitted use, you will need to obtain permission directly from the copyright holder. To view a copy of this license, visit http://creativecommons.org/ licenses/by/4.0/.

(C) The Author(s) 2020 